\title{
An Approach of Lebesgue Integral in Fuzzy Cone Metric Spaces via Unique Coupled Fixed Point Theorems
}

\author{
Muhammad Talha Waheed, ${ }^{1}$ Saif Ur Rehman ${ }^{(D,},{ }^{1}$ Naeem Jan ${ }^{D},{ }^{1}$ Abdu Gumaei ${ }^{D},{ }^{2}$ \\ and Mabrook Al-Rakhami ${ }^{3}{ }^{3}$ \\ ${ }^{1}$ Department of Mathematics, Gomal University, Dera Ismail Khan 29050, Pakistan \\ ${ }^{2}$ Computer Science Department, Faculty of Applied Sciences, Taiz University, Taiz 6803, Yemen \\ ${ }^{3}$ STC's Artificial Intelligence Chair, Department of Information Systems, College of Computer and Information Sciences, \\ King Saud University, Riyadh 11543, Saudi Arabia
}

Correspondence should be addressed to Naeem Jan; naeem.phdma73@iiu.edu.pk, Abdu Gumaei; abdugumaei@taiz.edu.ye, and Mabrook Al-Rakhami; malrakhami@ksu.edu.sa

Received 17 May 2021; Revised 4 July 2021; Accepted 2 August 2021; Published 17 August 2021

Academic Editor: Santosh Kumar

Copyright (c) 2021 Muhammad Talha Waheed et al. This is an open access article distributed under the Creative Commons Attribution License, which permits unrestricted use, distribution, and reproduction in any medium, provided the original work is properly cited.

\begin{abstract}
In the theory of fuzzy fixed point, many authors have been proved different contractive type fixed point results with different types of applications. In this paper, we establish some new fuzzy cone contractive type unique coupled fixed point theorems (FPtheorems) in fuzzy cone metric spaces (FCM-spaces) by using "the triangular property of fuzzy cone metric" and present illustrative examples to support our main work. In addition, we present a Lebesgue integral type mapping application to get the existence result of a unique coupled FP in FCM-spaces to validate our work.
\end{abstract}

\section{Introduction}

The theory of fuzzy sets was introduced by Zadeh [1]. Later on, in 1975, Kramosil and Michalek [2] introduced the concept of fuzzy metric spaces (FM-space); they present some structural properties of FM-space. In 1988, Grabiec [3] used the concept of Kramosil and Michalek [2] and proved two fixed point theorems (FP-theorems) of "Banach and Edelstein contraction mapping theorems on complete and compact FM-spaces, respectively." After that, the idea of FMspace given by Kramosil and Michalek [2] was modified by George and Veeramani [4], and they proved that every metric induces a fuzzy metric and also proved some fundamental properties and Baire's theorem for FM-spaces. In 2002, Gregory and Sapena [5] proved some contractive type FPtheorems in FM-spaces. Roldan et al. [6] presented some new FP-results in FM-spaces, while Jleli et al. [7] proved some results by using cyclic $(\psi, \phi)$-contractions in KalevaSeikkala's type fuzzy metric spaces. Kiany and Harandi [8] presented the concept of set-valued fuzzy-contractive type maps and proved some FP and end point results in FMspaces. Latterly, Rehman et al. [9] gave out the notion of rational type fuzzy contraction for FP in complete FMspaces with an application. Some more related FP-results can be found in [10-15].

Indeed, Huang and Zhang [16] rediscovered the idea of Banach-valued metric space. Indeed, many mathematicians proposed it; but it becomes popular after Huang and Zhang's study. By adopting the theory that the underlying cone is normal, they demonstrated the convergence properties and some FP-theorems. Rezapour and Hamlbarani [17], in 2008 , proved FP-theorems without assuming the cone's normality, while in [18] Karapinar proved some Ćirić-type nonunique FP-theorems on cone metric spaces. After that, many others contributed their ideas to the problem of FP-findings in cone metric spaces. A few of their FP-findings can be found (e.g., see [19-22]).

In 2015, Oner et al. [23] gave the idea of fuzzy cone metric space (FCM-space), and they also presented some fundamental properties and "a single-valued Banach contraction 
theorem for FP with the assumption that all the sequences are Cauchy." After that, Rehman and Li [24] settled some generalized fuzzy cone contractive type FP-results neglecting that "all the sequences are Cauchy" in complete FCM-space. And later, Jabeen et al. [25] presented some common FPtheorems for three self-mappings, by taking into consideration the idea of weakly compatible in FCM-spaces with an integral type application. Chen et al. [26], in 2020, gave the idea of coupled fuzzy cone contractive-type mappings. They proved "some coupled FP-theorems in FCM-spaces with non-linear integral type application." Latterly, Rehman and Aydi [27], in 2021, presented the concept of rational type fuzzy cone contraction mappings in FCM-spaces. They used "the triangular property of fuzzy metric" as a fundamental tool and proved some common FP-theorems and give an application.

Guo and Lakshmikantham [28] proved "coupled FPresults for the nonlinear operator with applications". Later, Bhaskar and Lakshmikantham [29] present some coupled FP-theorems in the context of partially ordered metric spaces, and this work is also presented by Lakshmikantham and Ciric [30]. In the year 2010, Sedghi et al. [31] proved some common coupled FP-results for commuting mappings in fuzzy metric spaces.

In this paper, we present some unique coupled FPfindings in FCM-spaces by taking the idea of Guo and Lakshmikantham [28] and Chen et al. [26]. Furthermore, we have also presented an application of the two Lebesgue Integral Equations (LIE) for a common solution to uphold our work. This paper is organized as follows: Section 2 consists of preliminaries. In Section 3, we establish some unique coupled FP-results in FCM-spaces with illustrative examples. In Section 4, we present an application of Lebesgue integral mapping to get the existence result of unique coupled FP in FCM-spaces to hold up our main work. In Section 5, we discuss the conclusion of our work.

\section{Preliminaries}

Definition 1 [32]. A binary operation $*:[0,1] \times[0,1] \longrightarrow[$ $0,1]$ would be a continuous $t$-norm if $*$ fulfils the following conditions:

(i) * is associative and commutative

(ii) $*$ is continuous

(iii) $1 * \alpha=\alpha, \forall \alpha \in[0,1]$

(iv) $\alpha_{1} * \alpha_{2} \leq \alpha_{3} * \alpha_{4}$ whenever $\alpha_{1} \leq \alpha_{3}$ and $\alpha_{2} \leq \alpha_{4}$, for $\alpha_{1}, \alpha_{2}, \alpha_{3}, \alpha_{4} \in[0,1]$

Throughout the complete paper, $\zeta$-norm represents a continuous $t$-norm.

Definition 2 [16]. Let $E$ be a real Banach space and $\vartheta$ be the zero element of $E$, and $C$ is a subset of $E$. Then, $C$ is called a cone if, (i) $C$ is closed and nonempty, and $C \neq\{\vartheta\}$

(ii) $\alpha_{1}, \alpha_{2} \in R, \alpha_{1}, \alpha_{2} \geq 0$ and $a, b \in C$, then $\alpha_{1} a+\alpha_{2} b \in C$

(iii) both $a \in C$ and $-a \in C$ and then $a=\vartheta$

A partial ordering on a given cone $C \subset E$ is defined by $a \preceq b \Leftrightarrow b-a \in$ C. $a \prec b$ stands for $a \leq b$ and $a \neq b$, while $a \ll$ $b$ stands for $b-a \in$ int $(C)$. In this paper, all cones have nonempty interior.

Definition 3 [4]. A 3-tuple $\left(A, \mathfrak{M}_{\mathfrak{c}}, *\right)$ is said to be a FMspace if $A$ is any set, $*$ is a $\zeta$-norm, and $\mathfrak{M}_{\text {c }}$ is a fuzzy set on $A^{2} \times(0, \infty)$ satisfying

(i) $\mathfrak{M}_{\mathfrak{c}}(a, b, \zeta)>0$

(ii) $\mathfrak{M}_{\mathfrak{c}}(a, b, \zeta)=0 \Leftrightarrow a=b$

(iii) $\mathfrak{M}_{\mathfrak{c}}(a, b, \zeta)=\mathfrak{M}_{\mathfrak{c}}(b, a, \zeta)$

(iv) $\mathfrak{M}_{\mathfrak{c}}(a, c, \zeta) * \mathfrak{M}_{\mathfrak{c}}(c, b, s) \leq \mathfrak{M}_{\mathfrak{c}}(a, b, \zeta+s)$

(v) $\mathfrak{M}_{\mathrm{c}}(a, b,):.(0, \infty) \longrightarrow[0,1]$ is tinuous, $\forall a, b, c \in A$ and $\zeta, s>0$

con-

Definition 4 [23]. A 3-tuple $\left(A, \mathfrak{M}_{\mathfrak{c}}, *\right)$ is said to be a FCMspace if $C$ is a cone of $E, A$ is an arbitrary set, $*$ is a $\zeta$-norm, and $\mathfrak{M}_{c}$ is a fuzzy set on $A^{2} \times$ int $(C)$ satisfying

(i) $\mathfrak{M}_{\mathfrak{c}}(a, b, \zeta)>0$

(ii) $\mathfrak{M}_{\mathrm{c}}(a, b, \zeta)=0 \Leftrightarrow a=b$

(iii) $\mathfrak{M}_{\mathfrak{c}}(a, b, \zeta)=\mathfrak{M}_{\mathfrak{c}}(b, a, \zeta)$

(iv) $\mathfrak{M}_{\mathfrak{c}}(a, c, \zeta) * \mathfrak{M}_{\mathfrak{c}}(c, b, s) \leq \mathfrak{M}_{\mathfrak{c}}(a, b, \zeta+s)$

(v) $\mathfrak{M}_{\mathfrak{c}}(a, b,):. \operatorname{int}(C) \longrightarrow[0,1]$ is continuous, $\forall a, b, c$ $\in A$, and $\zeta, s \gg \vartheta$

Definition 5 [23]. Let a 3-tuple $\left(A, \mathfrak{M}_{\mathfrak{c}}, *\right)$ be a FCM-space, $b_{1} \in A$, which is a sequence $\left\{b_{j}\right\}$ in $A$

(i) It converges to $b$ if $\alpha_{3} \in(0,1)$ and $\zeta \gg \vartheta$; there is $j_{1}$ $\in \mathcal{N}$ such that $\mathfrak{M}_{\mathfrak{c}}\left(b_{j}, b, \zeta\right)>1-\alpha_{3}$, for $j \geq j_{1}$, or we write it as $\lim _{j \longrightarrow \infty} b_{j}=b_{1}$ or $b_{j} \longrightarrow b_{1}$ as $j \longrightarrow \infty$

(ii) It is a Cauchy sequence if $\alpha_{3} \in(0,1)$ and $\zeta \gg 9$; there is $j_{1} \in \mathcal{N}$ such that $\mathfrak{M}_{\mathfrak{c}}\left(b_{j}, b_{i}, \zeta\right)>1-\alpha_{3}$, for $j, i \geq j_{1}$

(iii) $\left(A, \mathfrak{M}_{\mathfrak{c}}, *\right)$ is complete if every Cauchy sequence is convergent in $A$

(iv) It is fuzzy cone contractive if $\exists \alpha \in(0,1)$ and fulfilling

$$
\begin{gathered}
\frac{1}{\mathfrak{M}_{\mathfrak{c}}\left(b_{j}, b_{j+1}, \zeta\right)}-1 \leq \alpha\left(\frac{1}{\mathfrak{M}_{\mathfrak{c}}\left(b_{j-1}, b_{j}, \zeta\right)}-1\right) \\
\text { for } \zeta \gg \vartheta, j \geq 1 .
\end{gathered}
$$


Lemma 6 [23]. Let $\left(A, \mathfrak{M}_{\mathfrak{c}}, *\right)$ be a FCM-space, and let a sequence $\left\{b_{j}\right\}$ in $A$ converge to a point $b \in A \Leftrightarrow \mathfrak{M}_{\mathfrak{c}}\left(b_{j}, b, \zeta\right)$ which converges to 1 as $j \longrightarrow \infty$, for $\zeta \gg \vartheta$.

Definition 7 [24]. Let $\left(A, \mathfrak{M}_{\mathfrak{c}}, *\right)$ be a FCM-space. The FCM $\mathfrak{M}_{c}$ is triangular, if

$$
\begin{gathered}
\frac{1}{\mathfrak{M}_{\mathfrak{c}}(a, b, \zeta)}-1 \leq\left(\frac{1}{\mathfrak{M}_{\mathfrak{c}}(a, c, \zeta)}-1\right)+\left(\frac{1}{\mathfrak{M}_{\mathfrak{c}}(c, b, \zeta)}-1\right) \\
\forall a, c, b \in A, \zeta \gg \vartheta
\end{gathered}
$$

Definition 8 [23]. Let $\left(A, \mathfrak{M}_{\mathfrak{c}}, *\right)$ be a FCM-space and $\Gamma: A$ $\longrightarrow A$. Then, $\Gamma$ is said to be fuzzy cone contractive if $\exists$ $\alpha_{1} \in(0,1)$ such that

$\frac{1}{\mathfrak{M}_{\mathfrak{c}}(\Gamma b, \Gamma c, \zeta)}-1 \leq \alpha_{1}\left(\frac{1}{\mathfrak{M}_{\mathfrak{c}}(b, c, \zeta)}-1\right), \quad \forall b, c \in A, \zeta \gg \vartheta$

Definition 9. Let $(b, c)$ be an element in $A \times A$. Then, it is called coupled FP of a mapping $\Gamma: A \times A \longrightarrow A$ if

$$
\begin{aligned}
& \Gamma(b, c)=b, \\
& \Gamma(c, b)=c .
\end{aligned}
$$

Now, in the following, we prove some unique couple FPtheorems in FCM-spaces with examples to support our main work. Furthermore, we present an application of Lebesgue integral contractive type mapping to prove a unique coupled FP-theorem in FCM-spaces.

\section{Main Results}

Now, we present our first main result.

Theorem 10. Let $\Gamma: A \times A \longrightarrow A$ be a mapping on complete FCM-spaces $\left(A, \mathfrak{M}_{\mathfrak{c}}, *\right)$ in which $\mathfrak{M}_{\mathfrak{c}}$ is triangular and satisfies the inequality:

$$
\begin{aligned}
& \frac{1}{\mathfrak{M}_{\mathfrak{c}}(\Gamma(a, b), \Gamma(\kappa, \varrho), \zeta)}-1 \\
& \quad \leq \alpha_{1}\left(\frac{1}{\mathfrak{M}_{\mathfrak{c}}(a, \kappa, \zeta)}-1\right)+\alpha_{2}\left(\frac{1}{N(\Gamma,(a, b),(\kappa, \varrho), \zeta)}-1\right)
\end{aligned}
$$

where

$$
\begin{aligned}
& \frac{1}{N(\Gamma,(a, b),(\kappa, \varrho), \zeta)}-1 \\
& =\left(\frac{1}{\mathfrak{M}_{\mathfrak{c}}(a, \Gamma(a, b), \zeta)}-1+\frac{1}{\mathfrak{M}_{\mathfrak{c}}(\kappa, \Gamma(\kappa, \mathrm{Q}), \zeta)}\right. \\
& \left.-1+\frac{1}{\mathfrak{M}_{\mathfrak{c}}(a, \Gamma(\kappa, \mathrm{\varrho}), \zeta)}-1+\frac{1}{\mathfrak{M}_{\mathfrak{c}}(\kappa, \Gamma(a, b), \zeta)}-1\right)
\end{aligned}
$$

$\forall a, b, \kappa, \varrho \in A, \zeta \gg \vartheta, \alpha_{1} \in[0,1)$, and $\alpha_{2} \geq 0$ with $\left(\alpha_{1}+4 \alpha_{2}\right)$ $<1$. Then, $\Gamma$ has a unique couple FP in $A$.

Proof. Any $a_{0}, b_{0} \in A$; we define sequences $\left\{a_{j}\right\}$ and $\left\{b_{j}\right\}$ in $A$ such that

$$
\begin{gathered}
\Gamma\left(a_{j}, b_{j}\right)=a_{j+1}, \\
\Gamma\left(b_{j}, a_{j}\right)=b_{j+1}, \quad \text { for } j \geq 0 .
\end{gathered}
$$

Now from (5) for $\zeta \gg \vartheta$, we have

$$
\begin{aligned}
& \frac{1}{\mathfrak{M}_{\mathfrak{c}}\left(a_{j}, a_{j+1}, \zeta\right)}-1 \\
& =\frac{1}{\mathfrak{M}_{\mathfrak{c}}\left(\Gamma\left(a_{j-1}, b_{j-1}\right), \Gamma\left(a_{j}, b_{j}\right), \zeta\right)}-1 \\
& \quad \leq \alpha_{1}\left(\frac{1}{\mathfrak{M}_{\mathfrak{c}}\left(a_{j-1}, a_{j}, \zeta\right)}-1\right) \\
& \quad+\alpha_{2}\left(\frac{1}{N\left(\Gamma,\left(a_{j-1}, b_{j-1}\right),\left(a_{j}, b_{j}\right), \zeta\right)}-1\right),
\end{aligned}
$$

where

$$
\begin{aligned}
\frac{1}{N}( & \left.\Gamma,\left(a_{j-1}, b_{j-1}\right),\left(a_{j}, b_{j}\right), \zeta\right) \\
= & \left(\frac{1}{\mathfrak{M}_{\mathfrak{c}}\left(a_{j-1}, \Gamma\left(a_{j-1}, b_{j-1}\right), \zeta\right)}-1+\frac{1}{\mathfrak{M}_{\mathfrak{c}}\left(a_{j}, \Gamma\left(a_{j}, b_{j}\right), \zeta\right)}\right. \\
& -1+\frac{1}{\mathfrak{M}_{\mathfrak{c}}\left(a_{j-1}, \Gamma\left(a_{j}, b_{j}\right), \zeta\right)}-1 \\
& \left.+\frac{1}{\mathfrak{M}_{\mathfrak{c}}\left(a_{j}, \Gamma\left(a_{j-1}, b_{j-1}\right), \zeta\right)}-1\right) \\
= & \left(\frac{1}{\mathfrak{M}_{\mathfrak{c}}\left(a_{j-1}, a_{j}, \zeta\right)}-1+\frac{1}{\mathfrak{M}_{\mathfrak{c}}\left(a_{j}, a_{j+1}, \zeta\right)}\right. \\
& \left.-1+\frac{1}{\mathfrak{M}_{\mathfrak{c}}\left(a_{j-1}, a_{j+1}, \zeta\right)}-1\right) \\
\leq & 2\left(\frac{1}{\mathfrak{M}_{\mathfrak{c}}\left(a_{j-1}, a_{j}, \zeta\right)}-1+\frac{1}{\mathfrak{M}_{\mathfrak{c}}\left(a_{j}, a_{j+1}, \zeta\right)}-1\right)
\end{aligned}
$$


Now from (8) and (9), for $\zeta \gg \vartheta$,

$$
\begin{aligned}
& \frac{1}{\mathfrak{M}_{\mathfrak{c}}\left(a_{j}, a_{j+1}, \zeta\right)}-1 \\
& \leq \alpha\left(\frac{1}{\mathfrak{M}_{\mathfrak{c}}\left(a_{j-1}, a_{j}, \zeta\right)}-1\right)+2 \alpha_{2}\left(\frac{1}{\mathfrak{M}_{\mathfrak{c}}\left(a_{j-1}, a_{j}, \zeta\right)}\right. \\
& \left.\quad-1+\frac{1}{\mathfrak{M}_{\mathfrak{c}}\left(a_{j}, a_{j+1}, \zeta\right)}-1\right)
\end{aligned}
$$

We get, after simplification,

$$
\frac{1}{\mathfrak{M}_{\mathfrak{c}}\left(a_{j}, a_{j+1}, \zeta\right)}-1 \leq \lambda\left(\frac{1}{\mathfrak{M}_{\mathfrak{c}}\left(a_{j-1}, a_{j}, \zeta\right)}-1\right), \quad \text { for } \zeta \gg \vartheta
$$

where $\lambda=\left(\alpha_{1}+2 \alpha_{2}\right) /\left(1-2 \alpha_{2}\right)<1$. Similarly,

$\frac{1}{\mathfrak{M}_{\mathfrak{c}}\left(a_{j-1}, a_{j}, \zeta\right)}-1 \leq \lambda\left(\frac{1}{\mathfrak{M}_{\mathfrak{c}}\left(a_{j-2}, a_{j-1}, \zeta\right)}-1\right), \quad$ for $\zeta \gg \vartheta$

Now, from (11) and (12) and by induction, for $\zeta \gg \vartheta$,

$$
\begin{aligned}
& \frac{1}{\mathfrak{M}_{\mathfrak{c}}\left(a_{j}, a_{j+1}, \zeta\right)}-1 \\
& \quad \leq \lambda\left(\frac{1}{\mathfrak{M}_{\mathfrak{c}}\left(a_{j-1}, a_{j}, \zeta\right)}-1\right) \leq \lambda^{2}\left(\frac{1}{\mathfrak{M}_{\mathfrak{c}}\left(a_{j-2}, a_{j-1}, \zeta\right)}-1\right) \\
& \quad \leq \cdots \leq \lambda^{j}\left(\frac{1}{\mathfrak{M}_{\mathfrak{c}}\left(a_{0}, a_{1}, \zeta\right)}-1\right) \longrightarrow 0, \quad \text { as } j \longrightarrow \infty
\end{aligned}
$$

It shows that the sequence $\left\{a_{j}\right\}$ is a fuzzy cone contractive; therefore,

$$
\lim _{j \longrightarrow \infty} \mathfrak{M}_{\mathfrak{c}}\left(a_{j}, a_{j+1}, \zeta\right)=1, \quad \text { for } \zeta \gg \vartheta
$$

Now for $i>j$ and for $\zeta \gg \vartheta$, we have

$$
\begin{aligned}
& \frac{1}{\mathfrak{M}_{\mathfrak{c}}\left(a_{j}, a_{i}, \zeta\right)}-1 \\
& \leq\left(\frac{1}{\mathfrak{M}_{\mathfrak{c}}\left(a_{j}, a_{j+1}, \zeta\right)}-1\right)+\left(\frac{1}{\mathfrak{M}_{\mathfrak{c}}\left(a_{j+1}, a_{j+2}, \zeta\right)}-1\right) \\
& \quad+\cdots+\left(\frac{1}{\mathfrak{M}_{\mathfrak{c}}\left(a_{i-1}, a_{i}, \zeta\right)}-1\right)
\end{aligned}
$$

$$
\begin{aligned}
\leq & \lambda^{j}\left(\frac{1}{\mathfrak{M}_{\mathfrak{c}}\left(a_{o}, a_{1}, \zeta\right)}-1\right)+\lambda^{j+1}\left(\frac{1}{\mathfrak{M}_{\mathfrak{c}}\left(a_{0}, a_{1}, \zeta\right)}-1\right) \\
& +\cdots+\lambda^{i-1}\left(\frac{1}{\mathfrak{M}_{\mathfrak{c}}\left(a_{0}, a_{1}, \zeta\right)}-1\right) \\
= & \left(\lambda^{j}+\lambda^{j+1}+\cdots+\lambda^{i-1}\right)\left(\frac{1}{\mathfrak{M}_{\mathfrak{c}}\left(a_{0}, a_{1}, \zeta\right)}-1\right) \\
= & \frac{\lambda^{j}}{1-\lambda}\left(\frac{1}{\mathfrak{M}_{\mathfrak{c}}\left(a_{o}, a_{1}, \zeta\right)}-1\right) \longrightarrow 0, \quad \text { as } j \longrightarrow \infty .
\end{aligned}
$$

Hence, the sequence $\left\{a_{j}\right\}$ is Cauchy. Now for sequence $\left\{b_{j}\right\}$ and from (5), for $\zeta \gg \vartheta$, we have

$$
\begin{aligned}
& \frac{1}{\mathfrak{M}_{\mathfrak{c}}\left(b_{j}, b_{j+1}, \zeta\right)}-1 \\
& =\frac{1}{\mathfrak{M}_{\mathfrak{c}}\left(\Gamma\left(b_{j-1}, a_{j-1}\right), \Gamma\left(b_{j}, a_{j}\right), \zeta\right)}-1 \\
& \leq \alpha_{1}\left(\frac{1}{\mathfrak{M}_{\mathfrak{c}}\left(b_{j-1}, b_{j}, \zeta\right)}-1\right) \\
& +\alpha_{2}\left(\frac{1}{N\left(\Gamma,\left(b_{j-1}, a_{j-1}\right),\left(b_{j}, a_{j}\right), \zeta\right)}-1\right) \text {, }
\end{aligned}
$$

where

$$
\begin{aligned}
\frac{1}{N(} & \left.,\left(b_{j-1}, a_{j-1}\right),\left(b_{j}, a_{j}\right), \zeta\right) \\
= & \left(\frac{1}{\mathfrak{M}_{\mathfrak{c}}\left(b_{j-1}, \Gamma\left(b_{j-1}, a_{j-1}\right), \zeta\right)}-1\right. \\
& +\frac{1}{\mathfrak{M}_{\mathfrak{c}}\left(b_{j}, \Gamma\left(b_{j}, a_{j}\right), \zeta\right)} \\
& -1+\frac{1}{\mathfrak{M}_{\mathfrak{c}}\left(b_{j-1}, \Gamma\left(b_{j}, a_{j}\right), \zeta\right)}-1 \\
& \left.+\frac{1}{\mathfrak{M}_{\mathfrak{c}}\left(b_{j}, \Gamma\left(b_{j-1}, a_{j-1}\right), \zeta\right)}-1\right) \\
= & \left(\frac{1}{\mathfrak{M}_{\mathfrak{c}}\left(b_{j-1}, b_{j}, \zeta\right)}-1+\frac{1}{\mathfrak{M}_{\mathfrak{c}}\left(b_{j}, b_{j+1}, \zeta\right)}\right. \\
& \left.-1+\frac{1}{\mathfrak{M}_{\mathfrak{c}}\left(b_{j-1}, b_{j+1}, \zeta\right)}-1\right) \\
\leq & 2\left(\frac{1}{\mathfrak{M}_{\mathfrak{c}}\left(b_{j-1}, b_{j}, \zeta\right)}-1+\frac{1}{\mathfrak{M}_{\mathfrak{c}}\left(b_{j}, b_{j+1}, \zeta\right)}-1\right) .
\end{aligned}
$$

Now from (16) and (17), for $\zeta \gg \vartheta$, 


$$
\begin{aligned}
& \frac{1}{\mathfrak{M}_{\mathfrak{c}}\left(b_{j}, b_{j+1}, \zeta\right)}-1 \\
& \leq \alpha_{1}\left(\frac{1}{\mathfrak{M}_{\mathfrak{c}}\left(b_{j-1}, b_{j}, \zeta\right)}-1\right) \\
& \quad+2 \alpha_{2}\left(\frac{1}{\mathfrak{M}_{\mathfrak{c}}\left(b_{j-1}, b_{j}, \zeta\right)}-1+\frac{1}{\mathfrak{M}_{\mathfrak{c}}\left(b_{j}, b_{j+1}, \zeta\right)}-1\right)
\end{aligned}
$$

We get, after simplification,

$$
\frac{1}{\mathfrak{M}_{\mathfrak{c}}\left(b_{j}, b_{j+1}, \zeta\right)}-1 \leq \lambda\left(\frac{1}{\mathfrak{M}_{\mathfrak{c}}\left(b_{j-1}, b_{j}, \zeta\right)}-1\right), \quad \text { for } \zeta \gg \vartheta
$$

where $\lambda=\left(\alpha_{1}+2 \alpha_{2}\right) /\left(1-2 \alpha_{2}\right)<1$. Similarly,

$\frac{1}{\mathfrak{M}_{\mathfrak{c}}\left(b_{j-1}, b_{j}, \zeta\right)}-1 \leq \lambda\left(\frac{1}{\mathfrak{M}_{\mathfrak{c}}\left(b_{j-2}, b_{j-1}, \zeta\right)}-1\right), \quad$ for $\zeta \gg \vartheta$

Now, from (19) and (20) and by induction, for $\zeta \gg \vartheta$,

$$
\begin{aligned}
& \frac{1}{\mathfrak{M}_{\mathfrak{c}}\left(b_{j}, b_{j+1}, \zeta\right)}-1 \\
& \quad \leq \lambda\left(\frac{1}{\mathfrak{M}_{\mathfrak{c}}\left(b_{j-1}, b_{j}, \zeta\right)}-1\right) \leq \lambda^{2}\left(\frac{1}{\mathfrak{M}_{\mathfrak{c}}\left(b_{j-2}, b_{j-1}, \zeta\right)}-1\right) \\
& \quad \leq \cdots \leq \lambda^{j}\left(\frac{1}{\mathfrak{M}_{\mathfrak{c}}\left(b_{0}, b_{1}, \zeta\right)}-1\right) \longrightarrow 0, \text { as } j \longrightarrow \infty
\end{aligned}
$$

It shows that the sequence $\left\{b_{j}\right\}$ is a fuzzy cone contractive; therefore,

$$
\lim _{j \longrightarrow \infty} \mathfrak{M}_{\mathfrak{c}}\left(b_{j}, b_{j+1}, \zeta\right)=1, \quad \text { for } \zeta \gg \vartheta
$$

Now for $i>j$ and for $\zeta \gg \vartheta$, we have

$$
\begin{aligned}
& \frac{1}{\mathfrak{M}_{\mathfrak{c}}\left(b_{j}, b_{i}, \zeta\right)}-1 \\
& \leq\left(\frac{1}{\mathfrak{M}_{\mathfrak{c}}\left(b_{j}, b_{j+1}, \zeta\right)}-1\right)+\left(\frac{1}{\mathfrak{M}_{\mathfrak{c}}\left(b_{j+1}, b_{j+2}, \zeta\right)}-1\right) \\
& \quad+\cdots+\left(\frac{1}{\mathfrak{M}_{\mathfrak{c}}\left(b_{i-1}, h_{i}, \zeta\right)}-1\right)
\end{aligned}
$$

$$
\begin{aligned}
\leq & \lambda^{j}\left(\frac{1}{\mathfrak{M}_{\mathfrak{c}}\left(b_{o}, b_{1}, \zeta\right)}-1\right)+\lambda^{j+1}\left(\frac{1}{\mathfrak{M}_{\mathfrak{c}}\left(b_{0}, b_{1}, \zeta\right)}-1\right) \\
& +\cdots+\lambda^{i-1}\left(\frac{1}{\mathfrak{M}_{\mathfrak{c}}\left(b_{0}, b_{1}, \zeta\right)}-1\right) \\
= & \left(\lambda^{j}+\lambda^{j+1}+\cdots+\lambda^{i-1}\right)\left(\frac{1}{\mathfrak{M}_{\mathfrak{c}}\left(b_{0}, b_{1}, \zeta\right)}-1\right) \\
= & \frac{\lambda^{j}}{1-\lambda}\left(\frac{1}{\mathfrak{M}_{\mathfrak{c}}\left(b_{0}, b_{1}, \zeta\right)}-1\right) \longrightarrow 0, \quad \text { as } j \longrightarrow \infty .
\end{aligned}
$$

Hence, the sequence $\left\{b_{j}\right\}$ is Cauchy. Since $A$ is complete and $\left\{a_{j}\right\},\left\{b_{j}\right\}$ are Cauchy sequences in $A$, so $\exists a, b \in A$ such that $a_{j} \longrightarrow a$ and $b_{j} \longrightarrow b$ as $j \longrightarrow \infty$ or this can be written as $\lim _{j \longrightarrow \infty} a_{j}=a$ and $\lim _{j \longrightarrow \infty} b_{j}=b$. Therefore,

$$
\begin{gathered}
\lim _{j \longrightarrow \infty} \mathfrak{M}_{\mathfrak{c}}\left(a_{j}, a, \zeta\right)=1, \\
\lim _{j \longrightarrow \infty} \mathfrak{M}_{\mathfrak{c}}\left(b_{j}, b, \zeta\right)=1, \quad \text { for } \zeta \gg \vartheta .
\end{gathered}
$$

Hence,

$$
\begin{aligned}
\lim _{j \longrightarrow \infty} a_{j+1} & =\lim _{j \longrightarrow \infty} \Gamma\left(a_{j}, b_{j}\right)=\Gamma\left(\lim _{j \longrightarrow \infty} a_{j}, \lim _{j \longrightarrow \infty} b_{j}\right) \\
& \Rightarrow \Gamma(a, b)=a .
\end{aligned}
$$

Similarly,

$$
\begin{aligned}
\lim _{j \longrightarrow \infty} b_{j+1} & =\lim _{j \longrightarrow \infty} \Gamma\left(b_{j}, a_{j}\right)=\Gamma\left(\lim _{j \longrightarrow \infty} b_{j}, \lim _{j \longrightarrow \infty} a_{j}\right) \\
& \Rightarrow \Gamma(b, a)=b .
\end{aligned}
$$

Regarding its uniqueness, suppose $\left(a_{1}, b_{1}\right)$ and $\left(b_{1}, a_{1}\right)$ are another couple fixed point pairs in $A \times A$ such that $\Gamma$ ( $\left.a_{1}, b_{1}\right)=a_{1}$ and $\Gamma\left(b_{1}, a_{1}\right)=b_{1}$. Now, from (5), for $\zeta \gg \vartheta$, we have

$$
\begin{aligned}
\frac{1}{\mathfrak{M}_{\mathfrak{c}}\left(a, a_{1}, \zeta\right)}-1 \\
=\frac{1}{\mathfrak{M}_{\mathfrak{c}}\left(\Gamma(a, b), \Gamma\left(a_{1}, b_{1}\right), \zeta\right)}-1 \\
\leq \alpha_{1}\left(\frac{1}{\mathfrak{M}_{\mathfrak{c}}\left(a, a_{1}, \zeta\right)}-1\right) \\
\quad+\alpha_{2}\left(\frac{1}{N\left(\Gamma,(a, b),\left(a_{1}, b_{1}\right), \zeta\right)}-1\right),
\end{aligned}
$$

where 


$$
\begin{aligned}
& \frac{1}{N\left(\Gamma,(a, b),\left(a_{1}, b_{1}\right), \zeta\right)}-1 \\
& =\left(\frac{1}{\mathfrak{M}_{\mathfrak{c}}(a, \Gamma(a, b), \zeta)}-1+\frac{1}{\mathfrak{M}_{\mathfrak{c}}\left(a_{1}, \Gamma\left(a_{1}, b_{1}\right), \zeta\right)}-1\right. \\
& \left.+\frac{1}{\mathfrak{M}_{\mathfrak{c}}\left(a, \Gamma\left(a_{1}, b_{1}\right), \zeta\right)}-1+\frac{1}{\mathfrak{M}_{\mathfrak{c}}\left(a_{1}, \Gamma(a, b), \zeta\right)}-1\right) \\
& =\left(\frac{1}{\mathfrak{M}_{\mathfrak{c}}(a, a, \zeta)}-1+\frac{1}{\mathfrak{M}_{\mathfrak{c}}\left(a_{1}, a_{1}, \zeta\right)}-1+\frac{1}{\mathfrak{M}_{\mathfrak{c}}\left(a, a_{1}, \zeta\right)}\right. \\
& \left.-1+\frac{1}{\mathfrak{M}_{\mathfrak{c}}\left(a_{1}, a, \zeta\right)}-1\right)=2\left(\frac{1}{\mathfrak{M}_{\mathfrak{c}}\left(a, a_{1}, \zeta\right)}\right) .
\end{aligned}
$$

Now from (27) and for $\zeta \gg \vartheta$,

$$
\begin{aligned}
& \frac{1}{\mathfrak{M}_{\mathfrak{c}}\left(a, a_{1}, \zeta\right)}-1 \\
& \leq \alpha_{1}\left(\frac{1}{\mathfrak{M}_{\mathfrak{c}}\left(a, a_{1}, \zeta\right)}-1\right)+2 \alpha_{2}\left(\frac{1}{\mathfrak{M}_{\mathfrak{c}}\left(a, a_{1}, \zeta\right)}-1\right) \\
& =\left(\alpha_{1}+2 \alpha_{2}\right)\left(\frac{1}{\mathfrak{M}_{\mathfrak{c}}\left(a, a_{1}, \zeta\right)}-1\right) \\
& =\left(\alpha_{1}+2 \alpha_{2}\right)\left(\frac{1}{\mathfrak{M}_{\mathfrak{c}}\left(\Gamma(a, b), \Gamma\left(a_{1}, b_{1}\right), \zeta\right)}-1\right) \\
& \quad \leq\left(\alpha_{1}+2 \alpha_{2}\right)^{2}\left(\frac{1}{\mathfrak{M}_{\mathfrak{c}}\left(a, a_{1}, \zeta\right)}-1\right) \leq \cdots \\
& \quad \leq\left(\alpha_{1}+2 \alpha_{2}\right)^{j}\left(\frac{1}{\mathfrak{M}_{\mathfrak{c}}\left(a, a_{1}, \zeta\right)}-1\right) \\
& \quad \longrightarrow 0, \quad \text { as } j \longrightarrow \infty,
\end{aligned}
$$

where $\left(\alpha_{1}+2 \alpha_{2}\right)<1$. Hence, we have $\mathfrak{M}_{\mathfrak{c}}\left(a, a_{1}, \zeta\right)=1$ for $\zeta$ $\gg \vartheta, \Rightarrow a=a_{1}$.

Similarly, again from (5), for $\zeta \gg \vartheta$, we have

$$
\begin{aligned}
\frac{1}{\mathfrak{M}_{\mathfrak{c}}\left(b, b_{1}, \zeta\right)}-1= & \frac{1}{\mathfrak{M}_{\mathfrak{c}}\left(\Gamma(b, a), \Gamma\left(b_{1}, a_{1}\right), \zeta\right)}-1 \\
\leq & \alpha_{1}\left(\frac{1}{\mathfrak{M}_{\mathfrak{c}}\left(b, b_{1}, \zeta\right)}-1\right) \\
& \quad+\alpha_{2}\left(\frac{1}{N\left(\Gamma,(b, a),\left(b_{1}, a_{1}\right), \zeta\right)}-1\right),
\end{aligned}
$$

where

$$
\begin{aligned}
& \frac{1}{N\left(\Gamma,(b, a),\left(b_{1}, a_{1}\right), \zeta\right)}-1 \\
& \quad=\left(\frac{1}{\mathfrak{M}_{\mathfrak{c}}(b, \Gamma(b, a), \zeta)}-1+\frac{1}{\mathfrak{M}_{\mathfrak{c}}\left(b_{1}, \Gamma\left(b_{1}, a_{1}\right), \zeta\right)}-1\right. \\
& \left.\quad+\frac{1}{\mathfrak{M}_{\mathfrak{c}}\left(b, \Gamma\left(b_{1}, a_{1}\right), \zeta\right)}-1+\frac{1}{\mathfrak{M}_{\mathfrak{c}}\left(b_{1}, \Gamma(b, a), \zeta\right)}-1\right)
\end{aligned}
$$

$$
\begin{aligned}
= & \left(\frac{1}{\mathfrak{M}_{\mathfrak{c}}(b, b, \zeta)}-1+\frac{1}{\mathfrak{M}_{\mathfrak{c}}\left(b_{1}, b_{1}, \zeta\right)}-1+\frac{1}{\mathfrak{M}_{\mathfrak{c}}\left(b, b_{1}, \zeta\right)}\right. \\
& \left.-1+\frac{1}{\mathfrak{M}_{\mathfrak{c}}\left(b_{1}, b, \zeta\right)}-1\right)=2\left(\frac{1}{\mathfrak{M}_{\mathfrak{c}}\left(b, b_{1}, \zeta\right)}\right) .
\end{aligned}
$$

Now from (30) and for $\zeta \gg \vartheta$,

$$
\begin{aligned}
& \frac{1}{\mathfrak{M}_{\mathfrak{c}}\left(b, b_{1}, \zeta\right)}-1 \\
& \quad \leq \alpha_{1}\left(\frac{1}{\mathfrak{M}_{\mathfrak{c}}\left(b, b_{1}, \zeta\right)}-1\right)+2 \alpha_{2}\left(\frac{1}{\mathfrak{M}_{\mathfrak{c}}\left(b, b_{1}, \zeta\right)}-1\right) \\
& \quad=\left(\alpha_{1}+2 \alpha_{2}\right)\left(\frac{1}{\mathfrak{M}_{\mathfrak{c}}\left(b, b_{1}, \zeta\right)}-1\right) \\
& \quad=\left(\alpha_{1}+2 \alpha_{2}\right)\left(\frac{1}{\mathfrak{M}_{\mathfrak{c}}\left(\Gamma(b, a), \Gamma\left(b_{1}, a_{1}\right), \zeta\right)}-1\right) \\
& \quad \leq\left(\alpha_{1}+2 \alpha_{2}\right)^{2}\left(\frac{1}{\mathfrak{M}_{\mathfrak{c}}\left(b, b_{1}, \zeta\right)}-1\right) \leq \cdots \\
& \quad \leq\left(\alpha_{1}+2 \alpha_{2}\right)^{j}\left(\frac{1}{\mathfrak{M}_{\mathfrak{c}}\left(b, b_{1}, \zeta\right)}-1\right) \longrightarrow 0, \quad \text { as } j \longrightarrow \infty
\end{aligned}
$$

Hence, we have $\mathfrak{M}_{\mathfrak{c}}\left(b, b_{1}, \zeta\right)=1$ for $\zeta \gg \vartheta, \Rightarrow b=b_{1}$.

Corollary 11. Let $\Gamma: A \times A \longrightarrow A$ be a mapping on complete FCM-spaces $\left(A, \mathfrak{M}_{\mathfrak{c}}, *\right)$ in which $\mathfrak{M}_{\mathfrak{c}}$ is triangular and satisfies

$$
\begin{aligned}
& \frac{1}{\mathfrak{M}_{\mathfrak{c}}(\Gamma(a, b), \Gamma(\kappa, \varrho), \zeta)}-1 \\
& \leq \alpha_{1}\left(\frac{1}{\mathfrak{M}_{\mathfrak{c}}(a, \kappa, \zeta)}-1\right)+\alpha_{2}\left[\left(\frac{1}{\mathfrak{M}_{\mathfrak{c}}(a, \Gamma(a, b), \zeta)}-1\right)\right. \\
& \left.+\left(\frac{1}{\mathfrak{M}_{\mathfrak{c}}(\kappa, \Gamma(\kappa, \varrho), \zeta)}-1\right)\right],
\end{aligned}
$$

for all $a, b, \kappa, \varrho \in A, \zeta \gg \vartheta, \alpha_{1} \in[0,1)$, and $\alpha_{2} \geq 0$ with $\left(\alpha_{1}+\right.$ $\left.2 \alpha_{2}\right)<1$. Then, $\Gamma$ has a unique couple FP in $A$.

Corollary 12. Let $\Gamma: A \times A \longrightarrow A$ be a mapping on complete FCM-spaces $\left(A, \mathfrak{M}_{c}, *\right)$ in which $\mathfrak{M}_{c}$ is triangular and satisfies

$$
\begin{aligned}
\frac{1}{\mathfrak{M}_{\mathfrak{c}}(\Gamma(a, b), \Gamma(\kappa, \varrho), \zeta)}-1 \\
\leq \alpha_{1}\left(\frac{1}{\mathfrak{M}_{\mathfrak{c}}(a, \kappa, \zeta)}-1\right)+\alpha_{2}\left[\left(\frac{1}{\mathfrak{M}_{\mathfrak{c}}(a, \Gamma(\kappa, \varrho), \zeta)}-1\right)\right. \\
\left.\quad+\left(\frac{1}{\mathfrak{M}_{\mathfrak{c}}(\kappa, \Gamma(a, b), \zeta)}-1\right)\right]
\end{aligned}
$$


for all $a, b, \kappa, \mathrm{Q} \in A, \zeta \gg \vartheta, \alpha_{1} \in[0,1)$, and $\alpha_{2} \geq 0$ with $\left(\alpha_{1}+\right.$ $\left.2 \alpha_{2}\right)<1$. Then, $\Gamma$ has a unique couple FP in $A$.

Example 1. $A=(0, \infty), *$ is a $\zeta$-norm, and $\mathfrak{M}_{\mathfrak{c}}: A^{2} \times(0, \infty)$ $\longrightarrow[0,1]$ is defined as

$$
\mathfrak{M}_{\mathfrak{c}}(a, b, \zeta)=\frac{\zeta}{\zeta+d(a, b)}, \quad d(a, b)=|a-b|,
$$

for all $a, b \in A$ and $\zeta>0$. Then, it is easy to verify that $\mathfrak{M}_{\mathrm{c}}$ is triangular and $\left(A, \mathfrak{M}_{\mathfrak{c}}, *\right)$ is a complete $\mathrm{FCM}$-space. We define

$$
\Gamma(g, h)= \begin{cases}\frac{a-b}{12}, & \text { if } a, b \in[0,1] \\ \frac{2 a+2 b-2}{3}, & \text { if } a, b \in[1, \infty) .\end{cases}
$$

Now from (5), for $\zeta \gg \vartheta$, we have

$$
\begin{aligned}
& \frac{1}{\mathfrak{M}_{\mathfrak{c}}(\Gamma(a, b), \Gamma(\kappa, \mathrm{\varrho}), \zeta)}-1 \\
& =\left(\frac{1}{\mathfrak{M}_{\mathrm{c}}((\mathrm{a}-\mathrm{b}) / 12,(\kappa-\mathrm{e}) / 12, \zeta)}-1\right) \\
& =\frac{1}{\zeta}\left(d\left(\frac{a-b}{12}, \frac{\kappa-\mathrm{\varrho}}{12}\right)\right)=\frac{1}{12 \zeta}|a-b-\kappa+\mathrm{\varrho}| \\
& \leq \frac{1}{12 \zeta}[\mathrm{l}(a-\kappa)+(a-(a-b))+(\kappa-(\kappa-\mathrm{Q})) \\
& +(a-(\kappa-\mathrm{Q}))+(\kappa-(a-b)) \mid] \\
& \leq \frac{1}{12 \zeta}|a-\kappa|+\frac{1}{12 \zeta}(|a-(a-b)|+|\kappa-(\kappa-\mathrm{e})| \\
& +|a-(\kappa-\mathrm{e})|+|\kappa-(a-b)|) \\
& =\frac{1}{12}\left(\frac{1}{\mathfrak{M}_{\mathfrak{c}}(a, \kappa, \zeta)}-1\right)+\frac{1}{12}\left(\left(\frac{1}{\mathfrak{M}_{\mathfrak{c}}(a, \Gamma(a, b), \zeta)}-1\right)\right. \\
& +\left(\frac{1}{\mathfrak{M}_{\mathfrak{c}}(\kappa, \Gamma(\kappa, \mathrm{\varrho}), \zeta)}-1\right)+\left(\frac{1}{\mathfrak{M}_{\mathfrak{c}}(a, \Gamma(\kappa, \varrho), \zeta)}-1\right) \\
& \left.+\left(\frac{1}{\mathfrak{M}_{\mathfrak{c}}(\kappa, \Gamma(a, b), \zeta)}-1\right)\right)=\frac{1}{12}\left(\frac{1}{\mathfrak{M}_{\mathfrak{c}}(a, \kappa, \zeta)}-1\right) \\
& +\frac{1}{12}\left(\frac{1}{N(\Gamma,(a, b),(\kappa, \varrho), \zeta)}-1\right), \quad \text { for } \zeta \gg \vartheta
\end{aligned}
$$

It is easy to verify that conditions of Theorem 10 are satisfied with $\alpha_{1}=\alpha_{2}=1 / 12$. Then, $\Gamma$ has unique coupled FP for $a=2$ and $b=2$.

$$
\Gamma(a, b)=\Gamma(2,2)=\frac{2(2)+2(2)-2}{3}=2 \Rightarrow \Gamma(2,2)=2 .
$$

Theorem 13. Let $\Gamma: A \times A \longrightarrow A$ be a mapping in a complete FCM-space $\left(A, \mathfrak{M}_{\mathfrak{c}}, *\right)$ in which $\mathfrak{M}_{\mathfrak{c}}$ is triangular and satisfies

$$
\begin{aligned}
& \frac{1}{\mathfrak{M}_{\mathfrak{c}}(\Gamma(a, b), \Gamma(\kappa, \mathrm{Q}), \zeta)}-1 \\
& \leq \alpha_{1}\left(\frac{1}{\mathfrak{M}_{\mathfrak{c}}(a, \kappa, \zeta)}-1\right)+\alpha_{2}\left[\left(\frac{1}{\mathfrak{M}_{\mathfrak{c}}(a, \Gamma(a, b), \zeta)}-1\right)\right. \\
& \left.+\left(\frac{1}{\mathfrak{M}_{\mathfrak{c}}(\kappa, \Gamma(\kappa, \mathrm{Q}), \zeta)}-1\right)\right] \\
& +\alpha_{3}\left[\left(\frac{1}{\mathfrak{M}_{\mathfrak{c}}(\kappa, \Gamma(a, b), \zeta) * \mathfrak{M}_{\mathfrak{c}}(\kappa, \Gamma(\kappa, \mathrm{\varrho}), \zeta)}-1\right)\right],
\end{aligned}
$$

for all $a, b, \kappa, \varrho \in A, \zeta \gg \vartheta \alpha_{1} \in[0,1)$, and $\alpha_{2}, \alpha_{3} \geq 0$ with $\left(\alpha_{1}\right.$ $\left.+2 \alpha_{2}+\alpha_{3}\right)<1$. Then, $\Gamma$ has a unique couple FP in $A$.

Proof. Any $a_{0}, b_{0} \in A$, and we define sequence $\left\{a_{j}\right\}$ by

$$
\Gamma\left(a_{j}, b_{j}\right)=a_{j+1},
$$

$$
\Gamma\left(b_{j}, a_{j}\right)=b_{j+1}, \quad \text { for } j \geq 0 .
$$

Now, from (39), for $\zeta \gg$ 9, we have

$$
\begin{aligned}
\frac{1}{\mathfrak{M}_{\mathfrak{c}}\left(a_{j}, a_{j+1}, \zeta\right)}-1 \\
=\frac{1}{\mathfrak{M}_{\mathfrak{c}}\left(\Gamma\left(a_{j-1}, b_{j-1}\right), \Gamma\left(a_{j}, b_{j}\right), \zeta\right)}-1 \\
\leq \alpha_{1}\left(\frac{1}{\mathfrak{M}_{\mathfrak{c}}\left(a_{j-1}, a_{j}, \zeta\right)}-1\right) \\
\quad+\alpha_{2}\left[\left(\frac{1}{\mathfrak{M}_{\mathfrak{c}}\left(a_{j-1}, \Gamma\left(a_{j-1}, b_{j-1}\right), \zeta\right)}-1\right)\right. \\
\left.+\left(\frac{1}{\mathfrak{M}_{\mathfrak{c}}\left(a_{j}, \Gamma\left(a_{j}, b_{j}\right), \zeta\right)}-1\right)\right] \\
+\alpha_{3}\left(\frac{1}{\mathfrak{M}_{\mathfrak{c}}\left(a_{j}, \Gamma\left(a_{j-1}, b_{j-1}\right), \zeta\right) * \mathfrak{M}_{\mathfrak{c}}\left(a_{j}, \Gamma\left(a_{j}, b_{j}\right), \zeta\right)}-1\right) \\
=\alpha_{1}\left(\frac{1}{\mathfrak{M}_{\mathfrak{c}}\left(a_{j-1}, a_{j}, \zeta\right)}-1\right)+\alpha_{2}\left[\left(\frac{1}{\mathfrak{M}_{\mathfrak{c}}\left(a_{j-1}, a_{j}, \zeta\right)}-1\right)\right. \\
\left.+\left(\frac{1}{\mathfrak{M}_{\mathfrak{c}}\left(a_{j}, a_{j+1}, \zeta\right)}-1\right)\right] \\
+\alpha_{3}\left(\frac{1}{\mathfrak{M}_{\mathfrak{c}}\left(a_{j}, a_{j}, \zeta\right) * \mathfrak{M}_{\mathfrak{c}}\left(a_{j}, a_{j+1}, \zeta\right)}-1\right) \\
=\alpha_{1}\left(\frac{1}{\mathfrak{M}_{\mathfrak{c}}\left(a_{j-1}, a_{j}, \zeta\right)}-1\right)+\alpha_{2}\left[\left(\frac{1}{\left.\mathfrak{M}_{\mathfrak{c}}\left(a_{j-1}\right), a_{j}, \zeta\right)}-1\right)\right. \\
\left.+\left(\frac{1}{\mathfrak{M}_{\mathfrak{c}}\left(a_{j}, a_{j+1}, \zeta\right)}-1\right)\right]+\alpha_{3}\left(\frac{1}{\mathfrak{M}_{\mathfrak{c}}\left(a_{j}, a_{j+1}, \zeta\right)}-1\right) .
\end{aligned}
$$


We get, after simplification,

$\frac{1}{\mathfrak{M}_{\mathfrak{c}}\left(a_{j}, a_{j+1}, \zeta\right)}-1 \leq \delta\left(\frac{1}{\mathfrak{M}_{\mathfrak{c}}\left(a_{j-1}, a_{j}, \zeta\right)}-1\right), \quad$ for $\zeta \gg \vartheta$

where $\delta=\left(\alpha_{1}+\alpha_{2}\right) /\left(1-\alpha_{2}-\alpha_{3}\right)<1$. Similarly,

$\frac{1}{\mathfrak{M}_{\mathfrak{c}}\left(a_{j-1}, a_{j}, \zeta\right)}-1 \leq \delta\left(\frac{1}{\mathfrak{M}_{\mathfrak{c}}\left(a_{j-2}, a_{j-1}, \zeta\right)}-1\right), \quad$ for $\zeta \gg \vartheta$

Now, from (42) and (43) and by induction, for $\zeta \gg \vartheta$, we have

$$
\begin{aligned}
& \frac{1}{\mathfrak{M}_{\mathfrak{c}}\left(a_{j}, a_{j+1}, \zeta\right)}-1 \\
& \quad \leq \delta\left(\frac{1}{\mathfrak{M}_{\mathfrak{c}}\left(a_{j-1}, a_{j}, \zeta\right)}-1\right) \leq \delta^{2}\left(\frac{1}{\mathfrak{M}_{\mathfrak{c}}\left(a_{j-2}, a_{j-1}, \zeta\right)}-1\right) \\
& \quad \leq \cdots \leq \delta^{j}\left(\frac{1}{\mathfrak{M}_{\mathfrak{c}}\left(a_{o}, a_{1}, \zeta\right)}-1\right) \longrightarrow 0, \quad \text { as } j \longrightarrow \infty
\end{aligned}
$$

Hence, the sequence $\left\{a_{j}\right\}$ is fuzzy cone contractive; therefore,

$$
\lim _{j \longrightarrow \infty} \mathfrak{M}_{\mathfrak{c}}\left(a_{j}, a_{j+1}, \zeta\right)=1 \zeta \gg \vartheta
$$

Now for $i>j$ and for $\zeta \gg \vartheta$, we have

$$
\begin{aligned}
\frac{1}{\mathfrak{M}_{\mathfrak{c}}\left(a_{j}, a_{i}, \zeta\right)}-1 \\
\leq\left(\frac{1}{\mathfrak{M}_{\mathfrak{c}}\left(a_{j}, a_{j+1}, \zeta\right)}-1\right)+\left(\frac{1}{\mathfrak{M}_{\mathfrak{c}}\left(a_{j+1}, a_{j+2}, \zeta\right)}-1\right) \\
\quad+\cdots+\left(\frac{1}{\mathfrak{M}_{\mathfrak{c}}\left(a_{i-1}, a_{i}, \zeta\right)}-1\right) \\
\leq \delta^{j}\left(\frac{1}{\mathfrak{M}_{\mathfrak{c}}\left(a_{o}, a_{1}, \zeta\right)}-1\right)+\delta^{j+1}\left(\frac{1}{\mathfrak{M}_{\mathfrak{c}}\left(a_{o}, a_{1}, \zeta\right)}-1\right) \\
\quad+\cdots+\delta^{i-1}\left(\frac{1}{\mathfrak{M}_{\mathfrak{c}}\left(a_{o}, a_{1}, \zeta\right)}-1\right) \\
=\left(\delta^{j}+\delta^{j+1}+\cdots+\delta^{i-1}\right)\left(\frac{1}{\mathfrak{M}_{\mathfrak{c}}\left(a_{o}, a_{1}, \zeta\right)}-1\right) \\
=\frac{\delta^{j}}{1-\delta}\left(\frac{1}{\mathfrak{M}_{\mathfrak{c}}\left(a_{o}, a_{1}, \zeta\right)}-1\right) \longrightarrow 0, \text { as } j \longrightarrow \infty .
\end{aligned}
$$

Hence, the sequence $\left\{a_{j}\right\}$ is Cauchy. Now for sequence $\left\{b_{j}\right\}$, again from (39), for $\zeta \gg \vartheta$, we have

$$
\begin{aligned}
& \frac{1}{\mathfrak{M}_{\mathfrak{c}}\left(b_{j}, b_{j+1}, \zeta\right)}-1 \\
& =\frac{1}{\mathfrak{M}_{\mathfrak{c}}\left(\Gamma\left(b_{j-1}, a_{j-1}\right), \Gamma\left(b_{j}, a_{j}\right), \zeta\right)}-1 \\
& \leq \alpha_{1}\left(\frac{1}{\mathfrak{M}_{\mathfrak{c}}\left(b_{j-1}, b_{j}, \zeta\right)}-1\right) \\
& +\alpha_{2}\left[\left(\frac{1}{\mathfrak{M}_{\mathfrak{c}}\left(b_{j-1}, \Gamma\left(b_{j-1}, a_{j-1}, \zeta\right)\right.}-1\right)\right. \\
& \left.+\left(\frac{1}{\mathfrak{M}_{\mathfrak{c}}\left(b_{j}, \Gamma\left(b_{j}, a_{j}\right), \zeta\right)}-1\right)\right] \\
& +\alpha_{3}\left(\frac{1}{\mathfrak{M}_{\mathfrak{c}}\left(b_{j}, \Gamma\left(b_{j-1}, a_{j-1}\right), \zeta\right) * \mathfrak{M}_{\mathfrak{c}}\left(b_{j}, \Gamma\left(b_{j}, a_{j}\right), \zeta\right)}-1\right) \\
& =\alpha_{1}\left(\frac{1}{\mathfrak{M}_{\mathfrak{c}}\left(b_{j-1}, b_{j}, \zeta\right)}-1\right)+\alpha_{2}\left[\left(\frac{1}{\mathfrak{M}_{\mathfrak{c}}\left(b_{j-1}, b_{j}, \zeta\right)}-1\right)\right. \\
& \left.+\left(\frac{1}{\mathfrak{M}_{\mathfrak{c}}\left(b_{j}, b_{j+1}, \zeta\right)}-1\right)\right] \\
& +\alpha_{3}\left(\frac{1}{\mathfrak{M}_{\mathfrak{c}}\left(b_{j}, b_{j}, \zeta\right) * \mathfrak{M}_{\mathfrak{c}}\left(b_{j}, b_{j+1}, \zeta\right)}-1\right) \\
& =\alpha_{1}\left(\frac{1}{\mathfrak{M}_{\mathfrak{c}}\left(h_{j-1}, b_{j}, \zeta\right)}-1\right)+\alpha_{2}\left[\left(\frac{1}{\mathfrak{M}_{\mathfrak{c}}\left(b_{j-1}, b_{j}, \zeta\right)}-1\right)\right. \\
& \left.+\left(\frac{1}{\mathfrak{M}_{\mathfrak{c}}\left(b_{j}, b_{j+1}, \zeta\right)}-1\right)\right]+\alpha_{3}\left(\frac{1}{\mathfrak{M}_{\mathfrak{c}}\left(b_{j}, b_{j+1}, \zeta\right)}-1\right) .
\end{aligned}
$$

We get, after simplification,

$$
\frac{1}{\mathfrak{M}_{\mathfrak{c}}\left(b_{j}, b_{j+1}, \zeta\right)}-1 \leq \delta\left(\frac{1}{\mathfrak{M}_{\mathfrak{c}}\left(b_{j-1}, b_{j}, \zeta\right)}-1\right), \quad \text { for } \zeta \gg \vartheta
$$

where the value of $\delta$ is the same as in (42). Similarly,

$\frac{1}{\mathfrak{M}_{\mathfrak{c}}\left(b_{j-1}, b_{j}, \zeta\right)}-1 \leq \delta\left(\frac{1}{\mathfrak{M}_{\mathfrak{c}}\left(b_{j-2}, b_{j-1}, \zeta\right)}-1\right), \quad$ for $\zeta \gg \vartheta$

Now, from (48) and (49) and by induction, for $\zeta \gg 9$, we have that

$$
\begin{aligned}
& \frac{1}{\mathfrak{M}_{\mathfrak{c}}\left(b_{j}, b_{j+1}, \zeta\right)}-1 \\
& \quad \leq \delta\left(\frac{1}{\mathfrak{M}_{\mathfrak{c}}\left(b_{j-1}, b_{j}, \zeta\right)}-1\right) \leq \delta^{2}\left(\frac{1}{\mathfrak{M}_{\mathfrak{c}}\left(b_{j-2}, b_{j-1}, \zeta\right)}-1\right) \\
& \quad \leq \cdots \leq \delta^{j}\left(\frac{1}{\mathfrak{M}_{\mathfrak{c}}\left(b_{0}, b_{1}, \zeta\right)}-1\right) \longrightarrow 0, \quad \text { as } j \longrightarrow \infty .
\end{aligned}
$$


Hence, the sequence $\left\{b_{j}\right\}$ is fuzzy cone contractive; therefore,

$$
\lim _{j \longrightarrow \infty} \mathfrak{M}_{\mathfrak{c}}\left(b_{j}, b_{j+1}, \zeta\right)=1, \quad \text { for } \zeta \gg \vartheta
$$

Now for $i>j$, for $\zeta \gg \vartheta$, we have

$$
\begin{aligned}
\frac{1}{\mathfrak{M}_{\mathfrak{c}}\left(b_{j}, b_{i}, \zeta\right)}-1 \\
\leq\left(\frac{1}{\mathfrak{M}_{\mathfrak{c}}\left(b_{j}, b_{j+1}, \zeta\right)}-1\right)+\left(\frac{1}{\mathfrak{M}_{\mathfrak{c}}\left(b_{j+1}, b_{j+2}, \zeta\right)}-1\right) \\
\quad+\cdots+\left(\frac{1}{\mathfrak{M}_{\mathfrak{c}}\left(b_{i-1}, b_{i}, \zeta\right)}-1\right) \\
\leq \delta^{j}\left(\frac{1}{\mathfrak{M}_{\mathfrak{c}}\left(b_{0}, b_{1}, \zeta\right)}-1\right)+\delta^{j+1}\left(\frac{1}{\mathfrak{M}_{\mathfrak{c}}\left(b_{0}, b_{1}, \zeta\right)}-1\right) \\
\quad+\cdots+\delta^{i-1}\left(\frac{1}{\mathfrak{M}_{\mathfrak{c}}\left(b_{0}, b_{1}, \zeta\right)}-1\right) \\
=\left(\delta^{j}+\delta^{j+1}+\cdots+\delta^{i-1}\right)\left(\frac{1}{\mathfrak{M}_{\mathfrak{c}}\left(b_{0}, b_{1}, \zeta\right)}-1\right) \\
=\frac{\delta^{j}}{1-\delta}\left(\frac{1}{\mathfrak{M}_{\mathfrak{c}}\left(b_{0}, b_{1}, \zeta\right)}-1\right) \longrightarrow 0, \quad \text { as } j \longrightarrow \infty .
\end{aligned}
$$

Hence, the sequence $\left\{b_{j}\right\}$ is Cauchy. Since $A$ is complete and $\left\{a_{j}\right\}$ and $\left\{b_{j}\right\}$ are Cauchy sequences in $A, \exists a, b \in A$ such that $a_{j} \longrightarrow a$ and $b_{j} \longrightarrow b$ as $j \longrightarrow \infty$, or this can be written as $\lim _{j \longrightarrow \infty} a_{j}=a$ and $\lim _{j \longrightarrow \infty} b_{j}=b$. Therefore,

$$
\begin{aligned}
\lim _{j \longrightarrow \infty} a_{j+1} & =\lim _{j \longrightarrow \infty} \Gamma\left(a_{j}, b_{j}\right)=\Gamma\left(\lim _{j \longrightarrow \infty} a_{j}, \lim _{j \longrightarrow \infty} b_{j}\right) \\
& \Rightarrow \Gamma(a, b)=a .
\end{aligned}
$$

Similarly,

$$
\begin{aligned}
\lim _{j \longrightarrow \infty} b_{j+1} & =\lim _{j \longrightarrow \infty} \Gamma\left(b_{j}, a_{j}\right)=\Gamma\left(\lim _{j \longrightarrow \infty} b_{j}, \lim _{j \longrightarrow \infty} a_{j}\right) \\
& \Rightarrow \Gamma(b, a)=b .
\end{aligned}
$$

Regarding its uniqueness, let $\left(a_{1}, b_{1}\right)$ and $\left(b_{1}, a_{1}\right)$ be another couple fixed point pairs in $A \times A$ such that $\Gamma\left(a_{1}\right.$, $\left.b_{1}\right)=a_{1}$ and $\Gamma\left(b_{1}, a_{1}\right)=b_{1}$. Now, from (39), for $\zeta \gg \vartheta$, we have

$$
\begin{aligned}
& \frac{1}{\mathfrak{M}_{\mathfrak{c}}\left(a, a_{1}, \zeta\right)}-1 \\
= & \left(\frac{1}{\mathfrak{M}_{\mathfrak{c}}\left(\Gamma(a, b), \Gamma\left(a_{1}, b_{1}\right), \zeta\right)}-1\right) \\
\leq & \alpha_{1}\left(\frac{1}{\mathfrak{M}_{\mathfrak{c}}\left(a, a_{1}, \zeta\right)}-1\right)+\alpha_{2}\left[\left(\frac{1}{\mathfrak{M}_{\mathfrak{c}}(a, \Gamma(a, b), \zeta)-1}-1\right)\right. \\
& \left.+\left(\frac{1}{\mathfrak{M}_{\mathfrak{c}}\left(a_{1}, \Gamma\left(a_{1}, b_{1}\right), \zeta\right)}-1\right)\right] \\
& +\alpha_{3}\left(\frac{1}{\mathfrak{M}_{\mathfrak{c}}\left(a_{1}, \Gamma(a, b), \zeta\right) * \mathfrak{M}_{\mathfrak{c}}\left(a_{1}, \Gamma\left(a_{1}, b_{1}\right), \zeta\right)}-1\right) \\
= & \left(\alpha_{1}+\alpha_{3}\right)\left(\frac{1}{\mathfrak{M}_{\mathfrak{c}}\left(a, a_{1}, \zeta\right)}-1\right) \\
= & \left(\alpha_{1}+\alpha_{3}\right)\left(\frac{1}{\mathfrak{M}_{\mathfrak{c}}\left(\Gamma(a, b), \Gamma\left(a_{1}, b_{1}\right), \zeta\right)}-1\right) \\
\leq & \left(\alpha_{1}+\alpha_{3}\right)^{2}\left(\frac{1}{\mathfrak{M}_{\mathfrak{c}}\left(a, a_{1}, \zeta\right)}-1\right) \leq \cdots \\
\leq & \left(\alpha_{1}+\alpha_{3}\right)^{j}\left(\frac{1}{\mathfrak{M}_{\mathfrak{c}}\left(a, a_{1}, \zeta\right)}-1\right) \longrightarrow 0, \quad \text { as } j \longrightarrow \infty .
\end{aligned}
$$

Hence, we get that $\mathfrak{M}_{\mathfrak{c}}\left(a, a_{1}, \zeta\right)=1, \Rightarrow a=a_{1}$. Similarly, again from (39), for $\zeta \gg \vartheta$, we have

$$
\begin{aligned}
& \frac{1}{\mathfrak{M}_{\mathfrak{c}}\left(b, b_{1}, \zeta\right)}-1 \\
= & \left(\frac{1}{\mathfrak{M}_{\mathfrak{c}}\left(\Gamma(b, a), \Gamma\left(b_{1}, a_{1}\right), \zeta\right)}-1\right) \\
\leq & \alpha_{1}\left(\frac{1}{\mathfrak{M}_{\mathfrak{c}}\left(b, b_{1}, \zeta\right)}-1\right)+\alpha_{2}\left[\left(\frac{1}{\mathfrak{M}_{\mathfrak{c}}(b, \Gamma(b, a), \zeta)-1}-1\right)\right. \\
& \left.+\left(\frac{1}{\mathfrak{M}_{\mathfrak{c}}\left(b_{1}, \Gamma\left(b_{1}, a_{1}\right), \zeta\right)}-1\right)\right] \\
& +\alpha_{3}\left(\frac{1}{\mathfrak{M}_{\mathfrak{c}}\left(b_{1}, \Gamma(b, a), \zeta\right) * \mathfrak{M}_{\mathfrak{c}}\left(b_{1}, \Gamma\left(b_{1}, a_{1}\right), \zeta\right)}-1\right) \\
= & \left(\alpha_{1}+\alpha_{3}\right)\left(\frac{1}{\mathfrak{M}_{\mathfrak{c}}\left(b, b_{1}, \zeta\right)}-1\right) \\
= & \left(\alpha_{1}+\alpha_{3}\right)\left(\frac{1}{\mathfrak{M}_{\mathfrak{c}}\left(\Gamma(b, a), \Gamma\left(b_{1}, a_{1}\right), \zeta\right)}-1\right) \\
\leq & \left(\alpha_{1}+\alpha_{3}\right)^{2}\left(\frac{1}{\mathfrak{M}_{\mathfrak{c}}\left(b, b_{1}, \zeta\right)}-1\right) \leq \cdots \\
\leq & \left(\alpha_{1}+\alpha_{3}\right)^{j}\left(\frac{1}{\mathfrak{M}_{\mathfrak{c}}\left(b, b_{1}, \zeta\right)}-1\right) \longrightarrow 0, \quad \text { as } j \longrightarrow \infty .
\end{aligned}
$$

Hence, we get that $\mathfrak{M}_{\mathfrak{c}}\left(b, b_{1}, \zeta\right)=1$ for $\zeta \gg \vartheta, \Rightarrow b=b_{1}$.

Corollary 14. Let $\Gamma: A \times A \longrightarrow A$ be a mapping on complete FCM-spaces $\left(A, \mathfrak{M}_{\mathfrak{c}}, *\right)$ in which $\mathfrak{M}_{\mathfrak{c}}$ is triangular and satisfies 


$$
\begin{aligned}
& \frac{1}{\mathfrak{M}_{\mathfrak{c}}(\Gamma(a, b), \Gamma(\kappa, \varrho), \zeta)}-1 \\
& \leq \alpha_{1}\left(\frac{1}{\mathfrak{M}_{\mathfrak{c}}(a, \kappa, \zeta)}-1\right) \\
& +\alpha_{3}\left[\left(\frac{1}{\mathfrak{M}_{\mathfrak{c}}(\kappa, \Gamma(a, b), \zeta) * \mathfrak{M}_{\mathfrak{c}}(\kappa, \Gamma(\kappa, \varrho), \zeta)}-1\right)\right] \text {, }
\end{aligned}
$$

for all $a, b, \kappa, \varrho \in A, \zeta \gg \vartheta, \alpha_{1} \in(0,1)$, and $\alpha_{3} \geq 0$ with $\left(\alpha_{1}+\right.$ $\left.\alpha_{3}\right)<1$. Then, $\Gamma$ has a unique couple FP.

Example 2. $A=(0, \infty), *$ is a $\zeta$-norm, and $\mathfrak{M}_{c}: A \times A \times(0$, $\infty) \longrightarrow[0,1]$ is defined as

$$
\mathfrak{M}_{\mathfrak{c}}(a, b, \zeta)=\frac{\zeta}{\zeta+d(a, b)}, \quad d(a, b)=|a-b|
$$

for all $a, b \in A$ and $\zeta>0$. Then, it is easy to verify that $\mathfrak{M}_{c}$ is triangular and $\left(A, \mathfrak{M}_{\mathfrak{c}}, *\right)$ is a complete FCM-space. We define

$$
\ddot{F}(a, b)=\left\{\begin{array}{l}
\frac{a-b}{8}, \quad \text { if } a, b \in[0,1] \\
\frac{2 a+2 b-3}{3}, \quad \text { if } a, b \in[1, \infty) .
\end{array}\right.
$$

Now from (39), for $\zeta \gg \vartheta$, we have

$$
\begin{aligned}
& \frac{1}{\mathfrak{M}_{\mathfrak{c}}(\Gamma(a, b), \Gamma(\kappa, \varrho), \zeta)}-1 \\
& =\left(\frac{1}{\mathfrak{M}_{\mathfrak{c}}((a-b) / 8,(\kappa-\varrho) / 8, \zeta)}-1\right) \\
& =\frac{1}{\zeta}\left(d\left(\frac{a-b}{8}, \frac{\kappa-\varrho}{8}\right)\right)=\frac{1}{8 \zeta}(|a-b-\kappa+\varrho|) \\
& \leq \frac{1}{8 \zeta}[|a-\kappa|+|(a-(a-b))+(\kappa-(\kappa-\varrho))|] \\
& \leq \frac{1}{8 \zeta}|a-\kappa|+\frac{1}{8 \zeta}[|a-(a-b)|+|\kappa-(\kappa-\varrho)|] \\
& =\frac{1}{8}\left(\frac{1}{\mathfrak{M}_{\mathfrak{c}}(a, \kappa, \zeta)}-1\right)+\frac{1}{8}\left[\left(\frac{1}{\mathfrak{M}_{\mathfrak{c}}(a, \Gamma(a, b), \zeta)}-1\right)\right. \\
& \left.+\left(\frac{1}{\mathfrak{M}_{\mathfrak{c}}(\kappa, \Gamma(\kappa, \varrho), \zeta)}-1\right)\right], \quad \text { for } \zeta \gg \vartheta \text {. }
\end{aligned}
$$

It is easy to verify that all the conditions of Theorem 13 are satisfied with $\alpha_{1}=\alpha_{2}=1 / 8$ and $\alpha_{3}=0$. Then, $\Gamma$ has unique coupled FP.

$$
\Gamma(a, b)=\Gamma(3,3)=\frac{2(3)+2(3)-3}{3}=3 \Rightarrow \Gamma(3,3)=3 .
$$

\section{Application}

In this section, we present an application on Lebesgue integral (LI) mapping to support our main work. In 2002, Branciari proved the following result on complete metric space for unique FP (see [33]):

Theorem 15. Let $(A, d)$ be a complete metric space, $\alpha \in(0$, $1)$, and $\Gamma: A \longrightarrow A$ a mapping such that for each $a, b \in A$,

$$
\int_{0}^{d(\Gamma a, \Gamma b)} \varphi(s) d s \leq \alpha \int_{0}^{d(a, b)} \varphi(s) d s
$$

where $\varphi:[0, \infty) \longrightarrow[0, \infty)$ is a Lebesgue integrable mapping which is summable (i.e., with finite integral on each compact subset of $[0, \infty)$ ) and for each $\tau>0$,

$$
\int_{0}^{\tau} \varphi(s) d s>0
$$

Then, $\Gamma$ has a unique FP $u \in A$ such that for any $a \in A$, $\lim _{j \rightarrow \infty} \Gamma^{j} a=u$.

Now, we are in the position to use the above concept and to prove a unique coupled FP-theorem in FCM-spaces.

Theorem 16. Let $\Gamma: A \times A \longrightarrow A$ be a mapping on complete FCM-spaces $\left(A, \mathfrak{M}_{\mathfrak{c}}, *\right)$ in which $\mathfrak{M}_{\mathfrak{c}}$ is triangular and satisfies

$$
\begin{aligned}
& \int_{0}^{\left(\left(1 /\left(\mathfrak{M}_{\mathrm{c}}(\Gamma(a, b), \Gamma(\kappa, \mathfrak{e}), \zeta)\right)\right)-1\right)} \varphi(s) d s \\
& \quad \leq \alpha_{1} \int_{0}^{\left(\left(1 /\left(\mathfrak{M}_{\mathrm{c}}(a, \kappa, \zeta)\right)\right)-1\right)} \varphi(s) d s \\
& \quad+\alpha_{2} \int_{0}^{((1 /(N(\Gamma,(a, b),(\kappa, \mathrm{e}), \zeta)))-1)} \varphi(s) d s,
\end{aligned}
$$

where

$$
\begin{aligned}
& \frac{1}{N(\Gamma,(a, b),(\kappa, \mathrm{\varrho}), \zeta)}-1 \\
& \quad=\left(\frac{1}{\mathfrak{M}_{\mathfrak{c}}(a, \Gamma(a, b), \zeta)}-1+\frac{1}{\mathfrak{M}_{\mathfrak{c}}(\kappa, \Gamma(\kappa, \mathrm{\varrho}), \zeta)}-1\right. \\
& \left.\quad+\frac{1}{\mathfrak{M}_{\mathfrak{c}}(a, \Gamma(\kappa, \mathrm{\varrho}), \zeta)}-1+\frac{1}{\mathfrak{M}_{\mathfrak{c}}(\kappa, \Gamma(a, b), \zeta)}-1\right),
\end{aligned}
$$

for all $a, b, \kappa, \varrho \in A, \zeta \gg \vartheta, \alpha_{1} \in(0,1)$, and $\alpha_{2} \geq 0$ with $\left(\alpha_{1}+\right.$ $\left.4 \alpha_{2}\right)<1$ and $\varphi:[0, \infty) \longrightarrow[0, \infty)$ is a Lebesgue integrable mapping which is summable (i.e., with finite integral on each compact subset of $[0, \infty)$ ) and for each $\tau>0$,

$$
\int_{0}^{\tau} \varphi(s) d s>0
$$

Then, $\Gamma$ has a unique couple FP in $A$. 
Proof. Any $a_{o}, b_{o} \in A$; we define sequences $\left\{a_{j}\right\}$ and $\left\{b_{j}\right\}$ in $A$ such that

$$
\begin{gathered}
\Gamma\left(a_{j}, b_{j}\right)=a_{j+1}, \\
\Gamma\left(b_{j}, a_{j}\right)=b_{j+1}, \quad \text { for } j \geq 0 .
\end{gathered}
$$

Now from (64) and from the proof of Theorem 10, for $\zeta \gg \vartheta$, we have that

$$
\begin{aligned}
& \int_{0}\left(\left(1 /\left(\mathfrak{M}_{\mathrm{c}}\left(a_{j}, a_{j+1}, \zeta\right)\right)\right)-1\right) \varphi(s) d s \\
& \quad=\int_{0}^{\left(\left(1 /\left(\mathfrak{M}_{\mathrm{c}}\left(\Gamma\left(a_{j-1}, b_{j-1}\right), \Gamma\left(a_{j}, b_{j}\right), \zeta\right)\right)\right)-1\right)} \varphi(s) d s \\
& \quad \leq \lambda \int_{0}^{\left(\left(1 /\left(\mathfrak{M}_{\mathrm{c}}\left(a_{j-1}, a_{j}, \zeta\right)\right)\right)-1\right)} \varphi(s) d s,
\end{aligned}
$$

where $\lambda=\left(\alpha_{1}+2 \alpha_{2}\right) /\left(1-2 \alpha_{2}\right)<1$. Similarly, again by using the same arguments, we have

$$
\begin{aligned}
& \int_{0}^{\left(\left(1 /\left(\mathfrak{M}_{\mathrm{c}}\left(a_{j-1}, a_{j}, \zeta\right)\right)\right)-1\right)} \varphi(s) d s \\
& \quad \leq \lambda \int_{0}^{\left(\left(1 /\left(\mathfrak{M}_{\mathfrak{c}}\left(a_{j-2}, a_{j-1}, \zeta\right)\right)\right)-1\right)} \varphi(s) d s, \quad \text { for } \zeta \gg \vartheta .
\end{aligned}
$$
have

Now, from (68) and (69) and by induction, for $\zeta \gg \vartheta$, we

$$
\begin{aligned}
& \int_{0}^{\left(\left(1 /\left(\mathfrak{M}_{\mathrm{c}}\left(a_{j}, a_{j+1}, \zeta\right)\right)\right)-1\right)} \varphi(s) d s \\
& \quad \leq \lambda \int_{0}^{\left(\left(1 /\left(\mathfrak{M}_{\mathrm{c}}\left(a_{j-1}, a_{j}, \zeta\right)\right)\right)-1\right)} \varphi(s) d s \\
& \quad \leq \lambda^{2} \int_{0}^{\left(\left(1 /\left(\mathfrak{M}_{\mathfrak{c}}\left(a_{j-2}, a_{j-1}, \zeta\right)\right)\right)-1\right)} \varphi(s) d s \leq \cdots \\
& \quad \leq \lambda^{j} \int_{0}^{\left(\left(1 /\left(\mathfrak{M}_{\mathfrak{c}}\left(a_{0}, a_{1}, \zeta\right)\right)\right)-1\right)} \varphi(s) d s \longrightarrow 0, \quad \text { as } j \longrightarrow \infty,
\end{aligned}
$$

which shows that the sequence $\left\{a_{j}\right\}$ is a fuzzy cone contractive, therefore

$$
\begin{aligned}
& \lim _{j \longrightarrow \infty} \int_{0}^{\left(\left(1 /\left(\mathfrak{M}_{\mathfrak{c}}\left(a_{j}, a_{j+1}, \zeta\right)\right)\right)-1\right)} \varphi(s) d s \\
& \quad=0 \Rightarrow \lim _{j \longrightarrow \infty}\left(\frac{1}{\mathfrak{M}_{\mathfrak{c}}\left(a_{j}, a_{j+1}, \zeta\right)}-1\right) \\
& =0, \quad \text { for } \zeta \gg \vartheta .
\end{aligned}
$$

Hence, we get that

$$
\lim _{j \longrightarrow \infty} \mathfrak{M}_{\mathfrak{c}}\left(a_{j}, a_{j+1}, \zeta\right)=1, \quad \text { for } \zeta \gg \vartheta
$$

Now for $i>j$ and for $\zeta \gg \vartheta$, we have

$$
\begin{aligned}
& \int_{0}^{\left(\left(1 /\left(\mathfrak{M}_{\mathrm{c}}\left(a_{j}, a_{i}, \zeta\right)\right)\right)-1\right)} \varphi(s) d s \\
& \leq \int_{0}^{\left(\left(1 /\left(\mathfrak{M}_{\mathrm{c}}\left(a_{j}, a_{j+1}, \zeta\right)\right)\right)-1\right)} \varphi(s) d s \\
& +\int_{0}^{\left(\left(1 /\left(\mathfrak{M}_{\mathfrak{c}}\left(a_{j+1}, a_{j+2}, \zeta\right)\right)\right)-1\right)} \varphi(s) d s+\cdots \\
& +\int_{0}^{\left(\left(1 /\left(\mathfrak{M}_{\mathfrak{c}}\left(a_{i-1}, a_{i}, \zeta\right)\right)\right)-1\right)} \varphi(s) d s \\
& \leq \lambda^{j} \int_{0}^{\left(\left(1 /\left(\mathfrak{M}_{\mathrm{c}}\left(a_{o}, a_{1}, \zeta\right)\right)\right)-1\right)} \varphi(s) d s \\
& +\lambda^{j+1} \int_{0}^{\left(\left(1 /\left(\mathfrak{M}_{\mathrm{c}}\left(a_{o}, a_{1}, \zeta\right)\right)\right)-1\right)} \varphi(s) d s+\cdots \\
& +\lambda^{i-1} \int_{0}^{\left(\left(1 /\left(\mathfrak{M}_{\mathrm{c}}\left(a_{0}, a_{1}, \zeta\right)\right)\right)-1\right)} \varphi(s) d s \\
& =\left(\lambda^{j}+\lambda^{j+1}+\cdots+\lambda^{i-1}\right) \int_{0}^{\left(\left(1 /\left(\mathfrak{M}_{\mathrm{c}}\left(a_{0}, a_{1}, \zeta\right)\right)\right)-1\right)} \varphi(s) d s \\
& =\frac{\lambda^{j}}{1-\lambda} \int_{0}^{\left(\left(1 /\left(\mathfrak{M}_{\mathfrak{c}}\left(a_{0}, a_{1}, \zeta\right)\right)\right)-1\right)} \varphi(s) d s \\
& \longrightarrow 0, \quad \text { as } j \longrightarrow \infty \text {. }
\end{aligned}
$$

We get that

$$
\begin{aligned}
& \lim _{j \longrightarrow \infty} \int_{0}^{\left(\left(1 /\left(\mathfrak{M}_{\mathrm{c}}\left(a_{j}, a_{i}, \zeta\right)\right)\right)-1\right)} \varphi(s) d s \\
& \quad=0 \Rightarrow \lim _{j \longrightarrow \infty}\left(\frac{1}{\mathfrak{M}_{\mathfrak{c}}\left(a_{j}, a_{i}, \zeta\right)}-1\right)=0, \quad \text { for } \zeta \gg \vartheta
\end{aligned}
$$

Hence proved that the sequence $\left\{a_{j}\right\}$ is Cauchy. Now for sequence $\left\{b_{j}\right\}$ from (64) and from the proof of Theorem 10, for $\zeta \gg \vartheta$, we have

$$
\begin{aligned}
& \int_{0}^{\left(\left(1 /\left(\mathfrak{M}_{\mathfrak{c}}\left(b_{j}, b_{j+1}, \zeta\right)\right)\right)-1\right)} \varphi(s) d s \\
& \quad=\int_{0}^{\left(\left(1 /\left(\mathfrak{M}_{\mathfrak{c}}\left(\Gamma\left(b_{j-1}, a_{j-1}\right), \Gamma\left(b_{j}, a_{j}\right), \zeta\right)\right)\right)-1\right)} \varphi(s) d s \\
& \quad \leq \lambda \int_{0}^{\left(\left(1 /\left(\mathfrak{M}_{\mathfrak{c}}\left(b_{j-1}, b_{j}, \zeta\right)\right)\right)-1\right)} \varphi(s) d s,
\end{aligned}
$$

where $\lambda=\left(\alpha_{1}+2 \alpha_{2}\right) /\left(1-2 \alpha_{2}\right)<1$. Similarly, again by using the same arguments, we have

$$
\begin{aligned}
& \int_{0}^{\left(\left(1 /\left(\mathfrak{M}_{\mathfrak{c}}\left(b_{j-1}, b_{j}, \zeta\right)\right)\right)-1\right)} \varphi(s) d s \\
& \quad \leq \lambda \int_{0}^{\left(\left(1 /\left(\mathfrak{M}_{\mathfrak{c}}\left(b_{j-2}, a_{j-1}, \zeta\right)\right)\right)-1\right)} \varphi(s) d s, \quad \text { for } \zeta \gg \vartheta .
\end{aligned}
$$



have

Now, from (75) and (76) and by induction, for $\zeta \gg \vartheta$, we

$$
\begin{aligned}
& \int_{0}^{\left(\left(1 /\left(\mathfrak{M}_{\mathfrak{c}}\left(b_{j}, b_{j+1}, \zeta\right)\right)\right)-1\right)} \varphi(s) d s \\
& \quad \leq \lambda \int_{0}\left(\left(1 /\left(\mathfrak{M}_{\mathfrak{c}}\left(b_{j-1}, b_{j}, \zeta\right)\right)\right)-1\right) \varphi(s) d s \\
& \quad \leq \lambda^{2} \int_{0}^{\left(\left(1 /\left(\mathfrak{M}_{\mathfrak{c}}\left(b_{j-2}, b_{j-1}, \zeta\right)\right)\right)-1\right)} \varphi(s) d s \\
& \quad \leq \cdots \leq \lambda^{j} \int_{0}^{\left(\left(\left(1 /\left(\mathfrak{M}_{\mathfrak{c}}\left(b_{0}, b_{1}, \zeta\right)\right)\right)-1\right)\right.} \varphi(s) d s \\
& \quad \longrightarrow 0, \quad \text { as } j \longrightarrow \infty,
\end{aligned}
$$

which shows that the sequence $\left\{b_{j}\right\}$ is fuzzy cone contractive; therefore,

$$
\begin{aligned}
& \lim _{j \longrightarrow \infty} \int_{0}^{\left(\left(1 /\left(\mathfrak{M}_{\mathfrak{c}}\left(b_{j}, b_{j+1}, \zeta\right)\right)\right)-1\right)} \varphi(s) d s \\
& \quad=0 \Rightarrow \lim _{j \longrightarrow \infty}\left(\frac{1}{\mathfrak{M}_{\mathfrak{c}}\left(b_{j}, b_{j+1}, \zeta\right)}-1\right)=0, \quad \text { for } \zeta \gg \vartheta
\end{aligned}
$$

Hence, we get that

$$
\lim _{j \longrightarrow \infty} \mathfrak{M}_{\mathfrak{c}}\left(b_{j}, b_{j+1}, \zeta\right)=1, \quad \text { for } \zeta \gg \vartheta
$$

Now for $i>j$ and for $\zeta \gg \vartheta$, we have

$$
\begin{aligned}
& \int_{0}^{\left(\left(1 /\left(\mathfrak{M}_{\mathfrak{c}}\left(b_{j}, b_{i}, \zeta\right)\right)\right)-1\right)} \varphi(s) d s \\
& \leq \int_{0}^{\left(\left(1 /\left(\mathfrak{M}_{\mathfrak{c}}\left(b_{j}, b_{j+1}, \zeta\right)\right)\right)-1\right)} \varphi(s) d s \\
& \times+\int_{0}^{\left(\left(1 /\left(\mathfrak{M}_{\mathfrak{c}}\left(b_{j+1}, b_{j+2}, \zeta\right)\right)\right)-1\right)} \varphi(s) d s+\cdots \\
& +\int_{0}^{\left(\left(1 /\left(\mathfrak{M}_{\mathrm{c}}\left(b_{i-1}, b_{i}, \zeta\right)\right)\right)-1\right)} \varphi(s) d s \\
& \leq \lambda^{j} \int_{0}^{\left(\left(1 /\left(\mathfrak{M}_{\mathrm{c}}\left(b_{o}, b_{1}, \zeta\right)\right)\right)-1\right)} \varphi(s) d s \\
& +\lambda^{j+1} \int_{0}^{\left(\left(1 /\left(\mathfrak{M}_{\mathfrak{c}}\left(b_{o}, b_{1}, \zeta\right)\right)\right)-1\right)} \varphi(s) d s+\cdots \\
& +\lambda^{i-1} \int_{0}^{\left(\left(1 /\left(\mathfrak{M}_{\mathrm{c}}\left(b_{o}, b_{1}, \zeta\right)\right)\right)-1\right)} \varphi(s) d s \\
& =\left(\lambda^{j}+\lambda^{j+1}+\cdots+\lambda^{i-1}\right) \int_{0}^{\left(\left(1 /\left(\mathfrak{M}_{c}\left(b_{o}, b_{1}, \zeta\right)\right)\right)-1\right)} \varphi(s) d s \\
& =\frac{\lambda^{j}}{1-\lambda} \int_{0}^{\left(\left(1 /\left(\mathfrak{M}_{\mathrm{c}}\left(b_{o}, b_{1}, \zeta\right)\right)\right)-1\right)} \varphi(s) d s \longrightarrow 0, \quad \text { as } j \longrightarrow \infty \text {. }
\end{aligned}
$$

We get that

$$
\begin{aligned}
& \lim _{j \longrightarrow \infty} \int_{0}^{\left(\left(1 /\left(\mathfrak{M}_{\mathfrak{c}}\left(h_{j}, h_{i}, \zeta\right)\right)\right)-1\right)} \varphi(s) d s \\
& \quad=0 \Rightarrow \lim _{j \longrightarrow \infty}\left(\frac{1}{\mathfrak{M}_{\mathfrak{c}}\left(h_{j}, h_{i}, \zeta\right)}-1\right) \\
& \quad=0, \quad \text { for } \zeta \gg \vartheta .
\end{aligned}
$$

Hence, it was proved that the sequence $\left\{b_{j}\right\}$ is Cauchy. Since $A$ is complete and $\left\{a_{j}\right\},\left\{b_{j}\right\}$ are Cauchy sequences in $A$, so $\exists a, b \in A$ such that $a_{j} \longrightarrow a$ and $b_{j} \longrightarrow b$ as $j \longrightarrow$ $\infty$ or this can be written as $\lim _{j \longrightarrow \infty} a_{j}=a$ and $\lim _{j \longrightarrow \infty} b_{j}=b$. Therefore,

$$
\begin{gathered}
\lim _{j \longrightarrow \infty} \mathfrak{M}_{\mathfrak{c}}\left(a_{j}, a, \zeta\right)=1, \\
\lim _{j \longrightarrow \infty} \mathfrak{M}_{\mathfrak{c}}\left(b_{j}, b, \zeta\right)=1, \quad \text { for } \zeta \gg \vartheta .
\end{gathered}
$$

Hence,

$$
\begin{aligned}
\lim _{j \longrightarrow \infty} a_{j+1} & =\lim _{j \longrightarrow \infty} \Gamma\left(a_{j}, b_{j}\right)=\Gamma\left(\lim _{j \longrightarrow \infty} a_{j}, \lim _{j \longrightarrow \infty} b_{j}\right) \\
& \Rightarrow \Gamma(a, b)=a .
\end{aligned}
$$

Similarly,

$$
\begin{aligned}
\lim _{j \longrightarrow \infty} b_{j+1} & =\lim _{j \longrightarrow \infty} \Gamma\left(b_{j}, a_{j}\right)=\Gamma\left(\lim _{j \longrightarrow \infty} b_{j}, \lim _{j \longrightarrow \infty} a_{j}\right) \\
& \Rightarrow \Gamma(b, a)=b .
\end{aligned}
$$

Regarding its uniqueness, suppose $\left(a_{1}, b_{1}\right)$ and $\left(b_{1}, a_{1}\right)$ are another couple fixed point pairs in $A \times A$ such that $\Gamma$ ( $\left.a_{1}, b_{1}\right)=a_{1}$ and $\Gamma\left(b_{1}, a_{1}\right)=b_{1}$. Now, from (64) and from the proof of Theorem 10, for $\zeta \gg \vartheta$, we have that

$$
\begin{aligned}
& \int_{0}^{\left(\left(1 /\left(\mathfrak{M}_{\mathfrak{c}}\left(a, a_{1}, \zeta\right)\right)\right)-1\right)} \varphi(s) d s \\
& \quad=\int_{0}^{\left(\left(1 /\left(\mathfrak{M}_{\mathfrak{c}}\left(\Gamma(a, b), \Gamma\left(a_{1}, b_{1}\right), \zeta\right)\right)\right)-1\right)} \varphi(s) d s \\
& \quad \leq\left(\alpha_{1}+2 \alpha_{2}\right) \int_{0}^{\left(\left(1 /\left(\mathfrak{M}_{\mathfrak{c}}\left(a, a_{1}, \zeta\right)\right)\right)-1\right)} \varphi(s) d s \\
& \quad=\left(\alpha_{1}+2 \alpha_{2}\right) \int_{0}^{\left(\left(1 /\left(\mathfrak{M}_{\mathfrak{c}}\left(\Gamma(a, b), \Gamma\left(a_{1}, b_{1}\right), \zeta\right)\right)\right)-1\right)} \varphi(s) d s \\
& \quad \leq\left(\alpha_{1}+2 \alpha_{2}\right)^{2} \int_{0}^{\left(\left(1 /\left(\mathfrak{M}_{\mathfrak{c}}\left(a, a_{1}, \zeta\right)\right)\right)-1\right)} \varphi(s) d s \leq \cdots \\
& \quad \leq\left(\alpha_{1}+2 \alpha_{2}\right)^{j} \int_{0}^{\left(\left(1 /\left(\mathfrak{M}_{\mathfrak{c}}\left(a, a_{1}, \zeta\right)\right)\right)-1\right)} \varphi(s) d s \\
& \quad \longrightarrow 0, \quad \text { as } j \longrightarrow \infty .
\end{aligned}
$$

Hence, we get that $\mathfrak{M}_{\mathfrak{c}}\left(a, a_{1}, \zeta\right)=1$ for $\zeta \gg 9$; this implies $a=a_{1}$. 
Similarly, again from (64)and from the proof of Theorem 10 , for $\zeta \gg 9$, we have that

$$
\begin{aligned}
& \int_{0}^{\left(\left(1 /\left(\mathfrak{M}_{\mathfrak{c}}\left(b, b_{1}, \zeta\right)\right)\right)-1\right)} \varphi(s) d s \\
& \quad=\int_{0}^{\left(\left(1 /\left(\mathfrak{M}_{\mathfrak{c}}\left(\Gamma(b, a), \Gamma\left(b_{1}, a_{1}\right), t^{*}\right)\right)\right)-1\right)} \varphi(s) d s \\
& \quad \leq\left(\alpha_{1}+2 \alpha_{2}\right) \int_{0}^{\left(\left(1 /\left(\mathfrak{M}_{\mathfrak{c}}\left(b, b_{1}, \zeta\right)\right)\right)-1\right)} \varphi(s) d s \\
& \quad=\left(\alpha_{1}+2 \alpha_{2}\right) \int_{0}^{\left(\left(1 /\left(\mathfrak{M}_{\mathfrak{c}}\left(\Gamma(b, a), \Gamma\left(b_{1}, a_{1}\right), \zeta\right)\right)\right)-1\right)} \varphi(s) d s \\
& \quad \leq\left(\alpha_{1}+2 \alpha_{2}\right)^{2} \int_{0}^{\left(\left(1 /\left(\mathfrak{M}_{\mathfrak{c}}\left(b, b_{1}, \zeta\right)\right)\right)-1\right)} \varphi(s) d s \\
& \quad \leq \cdots \leq\left(\alpha_{1}+2 \alpha_{2}\right)^{j} \int_{0}^{\left(\left(\left(1 /\left(\mathfrak{M}_{\mathfrak{c}}\left(b, b_{1}, \zeta\right)\right)\right)-1\right)\right.} \varphi(s) d s \\
& \quad \longrightarrow 0, \quad \text { as } j \longrightarrow \infty .
\end{aligned}
$$
$b=b_{1}$.

Hence, we get that $\mathfrak{M}_{\mathfrak{c}}\left(b, b_{1}, \zeta\right)=1$ for $\zeta \gg 9$; this implies

\section{Conclusion}

We presented the concept of coupled FP-results in FCMspaces and prove some unique coupled FP-theorems under the modified contractive type conditions by using "the triangular property of fuzzy cone metric." We presented examples in support of our result. Further, we presented an application of Lebesgue integral mapping to uplift our main work. With the help of this new concept, one can prove more modified and general contractive type coupled FPresults with different types of integral contractive type of conditions and applications in complete FCM-spaces.

\section{Data Availability}

Data sharing is not applicable to this article as no data set was generated or analysed during the current study.

\section{Conflicts of Interest}

The authors declare that there is no conflict of interest regarding the publication of this paper.

\section{Acknowledgments}

The authors are grateful to the Deanship of Scientific Research, King Saud University for funding through Vice Deanship of Scientific Research Chairs.

\section{References}

[1] L. A. Zadeh, "Fuzzy Sets," Information and Control, vol. 8, no. 3, pp. 338-353, 1965.

[2] O. Kramosil and J. Michalek, "Fuzzy metric and statistical metric spaces," Kybernetika, vol. 11, pp. 336-344, 1975.
[3] M. Grabiec, "Fixed points in fuzzy metric spaces," Fuzzy Sets and Systems, vol. 27, no. 3, pp. 385-389, 1988.

[4] A. George and P. Veeramani, "On some results in fuzzy metric spaces,” Fuzzy Sets and Systems, vol. 64, no. 3, pp. 395-399, 1994.

[5] V. Gregori and A. Sapena, "On fixed-point theorems in fuzzy metric spaces," Fuzzy Sets and Systems, vol. 125, no. 2, pp. 245-252, 2002.

[6] A. Roldan, E. Karapinar, and S. Manro, "Some new fixed point theorems in fuzzy metric spaces," Journal of Intelligent Fuzzy Systems, vol. 27, no. 5, pp. 2257-2264, 2014.

[7] M. Jleli, E. Karapinar, and B. Samet, "On cyclic $(\psi$,$) -contrac-$ tions in Kaleva-Seikkala's type fuzzy metric spaces," Journal of Intelligent Fuzzy Systems, vol. 27, no. 4, pp. 2045-2053, 2014.

[8] F. Kiany and A. A. Harandi, "Fixed point and endpoint theorems for set-valued fuzzy contraction maps in fuzzy metric spaces," Fixed Point Theory and Applications, vol. 2011, 9 pages, 2011.

[9] S. U. Rehman, R. Chinram, and C. Boonpok, "Rational type fuzzy-contraction results in fuzzy metric spaces with an application," Journal of Mathematics, vol. 2021, 15 pages, 2021.

[10] C. D. Bari and C. Vetro, "Fixed points, attractors and weak fuzzy contractive mappings in a fuzzy metric space," Journal of Fuzzy Mathematics, vol. 1, pp. 973-982, 2005.

[11] X. Li, S. U. Rehman, S. U. Khan, H. Aydi, J. Ahmad, and N. Hussain, "Strong coupled fixed point results and applications to Urysohn integral equations," Dynamic Systems and Applications, vol. 30, pp. 197-218, 2021.

[12] B. D. Pant and S. Chauhan, "Common fixed point theorems for two pairs of weakly compatible mappings in Menger spaces and fuzzy metric spaces," Scientific Studies and Research, vol. 21, pp. 81-96, 2011.

[13] J. Rodriguez-Lopez and S. Romaguera, "The Haudorff fuzzy metric on compact sets," Fuzzy Sets and Systems, vol. 147, pp. 273-283, 2008.

[14] Z. Sadeghi, S. M. Vaezpour, C. Park, R. Saadati, and C. Vetro, "Set-valued mappings in partially ordered fuzzy metric spaces," Journal of Inequalities and Applications, vol. 2014, 17 pages, 2014.

[15] T. Som, "Some results on common fixed point in fuzzy metric spaces," Soochow Journal of Mathematics, vol. 33, pp. 553-561, 2007.

[16] L. Huang and X. Zhang, "Cone metric spaces and fixed point theorems of contractive mappings," Journal of Mathematical Analysis and Applications, vol. 332, no. 2, pp. 1468-1476, 2007.

[17] S. Rezapour and R. Hamlbarani, "Some notes on the paper "Cone metric spaces and fixed point theorems of contractive mappings"," Journal of Mathematical Analysis and Applications, vol. 345, no. 2, pp. 719-724, 2008.

[18] E. Karapinar, "Some nonunique fixed point theorems of Ćirić type on cone metric spaces," Abstract and Applied Analysis, vol. 2010, Article ID 123094, 14 pages, 2010.

[19] E. Karapinar, "Fixed point theorems in cone banach spaces," Fixed Point Theory and Applications, vol. 2009, no. 1, 2009.

[20] M. A. Khamsi, "Remarks on cone metric spaces and fixed point theorems of contractive mappings," Fixed Point Theory and Applications, vol. 2010, no. 1, 2010.

[21] G. Jungck and M. Abbas, "Common fixed point results for non-commuting mappings without continuity in cone metric 
spaces," Journal of Mathematical Analysis and Applications, vol. 341, p. 416, 2008.

[22] V. Rakovcevic and D. Ilic, "Common fixed points for maps on cone metric spaces," Journal of Mathematical Analysis and Applications, vol. 341, 2008.

[23] T. Oner, M. B. Kandemire, and B. Tanay, "Fuzzy cone metric spaces," Journal of Nonlinear Sciences and Applications, vol. 8, no. 5, pp. 610-616, 2015.

[24] S. U. Rehman and H. X. Li, "Fixed point theorems in fuzzy cone metric spaces," Journal of Nonlinear Sciences and Applications, vol. 10, no. 11, pp. 5763-5769, 2017.

[25] S. Jabeen, S. U. Rehman, Z. Zheng, and W. Wei, "Weakly compatible and quasi-contraction results in fuzzy cone metric spaces with application to the Urysohn type integral equations," Advances in Differential Equations, vol. 2020, 2020.

[26] G. X. Chen, S. Jabeen, S. U. Rehman et al., "Coupled fixed point analysis in fuzzy cone metric spaces with an application to nonlinear integral equations," Advances in Differential Equations, vol. 2020, no. 1, p. 671, 2020.

[27] S. U. Rehman and H. Aydi, "Rational fuzzy cone contractions on fuzzy cone metric spaces with an application to Fredholm integral equations," Journal of Function Spaces, vol. 2021, Article ID 5527864, 13 pages, 2021.

[28] D. Guo and V. Lakshmikantham, "Coupled fixed points of nonlinear operators with applications," Nonlinear Analysis, vol. 11, no. 5, pp. 623-632, 1987.

[29] T. G. Bhaskar and V. Lakshmikantham, "Fixed point theorems in partially ordered metric spaces and applications," Nonlinear analysis: theory, methods \& applications, vol. 65, no. 7, pp. 1379-1393, 2006.

[30] V. Lakshmikantham and L. Ciric, "Coupled fixed point theorems for nonlinear contractions in partially ordered metric spaces," Nonlinear analysis: theory, methods \& applications, vol. 70, no. 12, pp. 4341-4349, 2009.

[31] S. Sedghi, I. Altun, and N. Shobe, "Coupled fixed point theorems for contractions in fuzzy metric spaces," Nonlinear Analysis, vol. 72, no. 3-4, pp. 1298-1304, 2010.

[32] B. Schweizer and A. Sklar, Probabilistic Metric Spaces, NorthHolland Series in Probability and Applied Mathematics, New York, USA, 1983.

[33] A. Branciari, "A fixed point theorem for mappings satisfying a general contractive condition of integral type," International Journal of Mathematics and Mathematical Sciences, vol. 29, no. 9, 536 pages, 2002. 